\title{
STRONG AND FULL DUALISABILITY: THREE-ELEMENT UNARY ALGEBRAS
}

\author{
J. G. PITKETHLY
}

(Received 30 May 2000; revised 21 September 2001)

Communicated by C. F. Miller

\begin{abstract}
We characterise the strongly dualisable three-element unary algebras and show that every fully dualisable three-element unary algebra is strongly dualisable. It follows from the characterisation that, for dualisable three-element unary algebras, strong dualisability is equivalent to a weak form of injectivity.
\end{abstract}

2000 Mathematics subject classification: primary 08C15, 08A60; secondary 18A40.

Keywords and phrases: natural duality, strong duality, full duality, unary algebras.

The theory of natural dualities is a study of quasi-varieties of the form $\mathbb{D S P}(\underline{\mathbf{M}})$, where $\underline{\mathbf{M}}$ is a finite algebra. We aim to set up a natural dual equivalence between the category $\mathscr{A}:=\mathbb{1 S P}(\underline{\mathbf{M}})$ and a category $\mathscr{X}$ of structured topological spaces. This duality can often provide a practical representation of the algebras in $\mathscr{A}$ in terms of simpler objects. Priestley's duality for the quasi-variety of distributive lattices is a prime example of a very useful duality (see [9]). As well as finding and using practical dualities, natural-duality theoreticians tackle more esoteric problems. We are interested in understanding which finite algebras $\underline{\mathbf{M}}$ allow us to set up a natural duality for $\mathbb{\triangle S P}(\mathbf{M})$, and what the existence (or non-existence) of this duality can tell us about the quasi-variety $\mathbb{\triangle S P ( M )}$.

The theory of natural dualities is well developed and contains some powerful theorems for creating dualities. Nevertheless, our understanding of what makes an algebra dualisable, fully dualisable or strongly dualisable is rather limited. In this paper, we aim to gain some insight into strong and full dualisability by investigating threeelement unary algebras. Unary algebras, especially three-element unary algebras, may seem very simple. But, from the point of view of natural-duality theory, they are rather complicated. This study complements the paper [4], by Clark, Davey and 
Pitkethly, which classifies the dualisable three-element unary algebras. We give a characterisation of the strongly and fully dualisable three-element unary algebras. In particular, we show that strong and full dualisability are equivalent within this class. In general, it is not known whether every fully dualisable algebra is strongly dualisable. The strong dualisability of an algebra seems to depend on how close the algebra is to being injective. We show that, for dualisable three-element unary algebras, strong dualisability is equivalent to a weak form of injectivity. In [7], Hyndman and Willard give an example of a three-element unary algebra that is dualisable but not fully dualisable. It follows from our characterisation that there are many three-element unary algebras that are dualisable but not fully dualisable.

The (strong) dualisability of a three-element unary algebra is related to the number of different patterns of its unary term functions. Consider a finite unary algebra $\underline{\mathbf{M}}$. We shall define a kernel of $\underline{\mathbf{M}}$ to be an equivalence relation on $M$ that is the kernel of a unary term function of $\underline{M}$ which is not a constant map or a permutation. We call $\underline{M}$ an $n$-kernel unary algebra if $n$ is the number of kernels of $\underline{M}$.

The following theorem gives the classification of dualisable three-element unary algebras from [4]. This classification is most complicated within the family of twokernel algebras. To simplify the statement of the theorem, we use the fact that every two-kernel three-element unary algebra is isomorphic to a unary algebra, on the set $\{0,1,2\}$, with kernels $\{01 \mid 2\}$ and $\{02 \mid 1\}$. (See Lemma 4.1 [4].) We denote a unary operation $u:\{0,1,2\} \rightarrow\{0,1,2\}$ by the string $u(0) u(1) u(2)$.

DUALISABLE THREE-ELEMENT UNARY ALGEBRAS. Let $\underline{\mathbf{M}}$ be a three-element unary algebra.

(i) If $\underline{\mathbf{M}}$ is a zero-kernel or one-kernel algebra, then $\underline{\mathbf{M}}$ is dualisable.

(ii) Assume that $\underline{M}$ is a two-kernel algebra, on the set $\{0,1,2\}$, with kernels $\{01 \mid 2\}$ and $\{02 \mid 1\}$. Then $\underline{\mathrm{M}}$ is dualisable if and only if none of the following conditions hold:

(a) $p p q$ and $p q p$ are term functions of $\underline{\mathrm{M}}$, for some $p, q \in M$ with $p \neq q$, but 010 or 002 is not a term function of $\underline{\mathbf{M}}$;

(b) 010,001 and 110 are term functions of $\underline{\mathbf{M}}$, but 222 isn't;

(c) 002, 020 and 202 are term functions of $\underline{\mathrm{M}}$, but 111 isn't.

(iii) If $\underline{\mathrm{M}}$ is a three-kernel algebra, then $\underline{\mathrm{M}}$ is not dualisable.

In this paper, we shall establish the characterisations of strongly and fully dualisable three-element unary algebras given below.

STRONGLY DUALISABLE THREE-ELEMENT UNARY ALGEBRAS. Let $\underline{\mathbf{M}}$ be a threeelement unary algebra.

(i) If $\underline{\mathbf{M}}$ is a zero-kernel or one-kernel algebra, then $\underline{\mathbf{M}}$ is strongly dualisable. 
(ii) Assume that $\underline{\mathbf{M}}$ is a two-kernel algebra, on the set $\{0,1,2\}$, with kernels $\{01 \mid 2\}$ and $\{02 \mid 1\}$. Then $\underline{\mathbf{M}}$ is strongly dualisable if and only if both 010 and 002 are term functions of $\underline{\mathrm{M}}$ and neither of the following conditions holds:

(a) $p p q$ and $q p q$ are term functions of $\underline{\mathbf{M}}$, for some $p, q \in M$ with $p \neq q$;

(b) 101 and 220 are term functions of $\underline{\mathbf{M}}$.

(iii) If $\underline{\mathrm{M}}$ is a three-kernel algebra, then $\underline{\mathrm{M}}$ is not strongly dualisable.

Explicit examples of strongly dualisable two-kernel three-element unary algebras may be obtained from Lemma 3.6.

FULLY DUALISABLE THREE-ELEMENT UNARY ALGEBRAS. Let $\underline{\mathbf{M}}$ be a three-element unary algebra. Then $\underline{\mathbf{M}}$ is fully dualisable if and only if $\underline{\mathbf{M}}$ is strongly dualisable.

We have given an algorithm for deciding whether or not a particular three-element unary algebra is strongly dualisable. However, this algorithm does not really give us a feel for what makes a three-element unary algebra strongly dualisable. We shall show that, for dualisable three-element unary algebras, strong dualisability is equivalent to a weak form of injectivity. To make this more precise, we first need to give some definitions. (Many of the concepts we use throughout this paper are introduced in more detail, and with examples, in the paper [4].)

Let $\underline{M}$ be a finite unary algebra and choose an algebra $\mathbf{A}$ from the quasi-variety $\mathscr{A}:=\operatorname{ISP}(\mathbf{M})$. The centre of $\mathbf{A}$ is defined to be the subuniverse

$$
C_{\mathbf{A}}:=\left\{m^{\mathbf{A}} \mid m \in M \text { is the value of a constant term function of } \underline{\mathbf{M}}\right\}
$$

of $\mathbf{A}$. There is a directed graph naturally associated with $\mathbf{A}$. We define the graph $G(\mathrm{~A})=\left\langle A ; E_{\mathrm{A}}\right\rangle$ by

$$
E_{\mathrm{A}}:=\left\{(a, b) \mid a \in A \text { and } b \in \operatorname{sg}_{\mathrm{A}}(a)\right\},
$$

where $\operatorname{sg}_{\mathbf{A}}(a)$ denotes the subuniverse of $\mathbf{A}$ generated by $a$, for each $a \in A$. The relation $E_{\mathrm{A}}$ is reflexive and transitive, and so is a quasi-order on $A$. Let $G^{*}(\mathbf{A})$ denote the induced subgraph of $G(\mathrm{~A})$ with vertex set $A \backslash C_{\mathrm{A}}$. For every $a, b \in A \backslash C_{\mathrm{A}}$ and $n \in \mathbb{N} \cup\{0\}$, we say that there is a fence from $a$ to $b$ in $\mathbf{A}$ of length $n$ if there are edges $x_{1}, y_{1}, \ldots, x_{n}, y_{n}$ of $G^{*}(\mathbf{A})$ such that

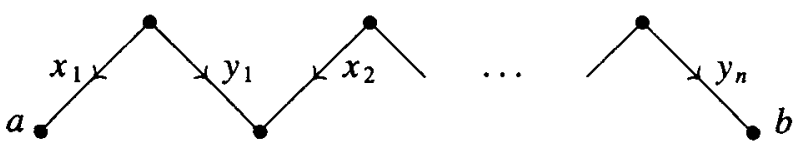

in $G^{*}(\mathbf{A})$. A subalgebra $\mathbf{P}$ of $\mathbf{A}$ is called a petal of $\mathbf{A}$ if $P \backslash C_{\mathbf{A}}$ is the vertex set of a connected component of the graph $G^{*}(\mathrm{~A})$. So $a, b \in A \backslash C_{\mathrm{A}}$ belong to the same petal 
of $\mathbf{A}$ if and only if there is a fence from $a$ to $b$ in $\mathbf{A}$. It is easy to check that every non-trivial algebra in $\mathscr{A}$ is the coproduct of its petals.

Now let $\mathbf{P}$ be a petal of $\mathbf{A}$ and let $n \in \mathbb{N}$. Define the distance function $d_{\mathbf{P}}$ on $P \backslash C_{\mathrm{A}}$ such that $d_{\mathrm{P}}(a, b)$ is the length of the shortest fence from $a$ to $b$ in $\mathbf{A}$, for all $a, b \in P \backslash C_{\mathrm{A}}$. For each $a \in P \backslash C_{\mathrm{A}}$, the ball in $\mathbf{A}$ with centre $a$ and radius $n$, given by

$$
n_{\mathbf{A}}(a):=\left\{b \in P \backslash C_{\mathbf{A}} \mid d_{\mathbf{P}}(a, b) \leqslant n\right\} \cup C_{\mathbf{A}},
$$

determines a subalgebra of $\mathrm{A}$. For $a \in C_{\mathrm{A}}$, we set $n_{\mathrm{A}}(a):=C_{\mathrm{A}}$. Now define $n_{\mathrm{A}}(B):=\bigcup\left\{n_{\mathrm{A}}(b) \mid b \in B\right\}$, for all $B \subseteq A$. The algebra $\underline{\mathbf{M}}$ is said to be $n$-quasiinjective if, for all finite algebras $\mathbf{A}, \mathbf{B} \in \mathscr{A}$ such that $\mathbf{B} \leqslant \mathbf{A}$, every homomorphism $x: \mathbf{B} \rightarrow \underline{M}$ that extends to $n_{\mathrm{A}}(B)$ also extends to $A$. We shall show that the theorem below follows from our description of strongly dualisable three-element unary algebras.

QUASI-INJECTIVITY THEOREM. Let $\underline{\mathbf{M}}$ be a dualisable three-element unary algebra. Then $\underline{\mathbf{M}}$ is strongly dualisable if and only if $\underline{\mathbf{M}}$ is n-quasi-injective, for some $n \in \mathbb{N}$.

\section{Natural dualities}

This section provides a quick introduction to duality theory. A more detailed account can be found in the text [1], by Clark and Davey. Let $\underline{\mathbf{M}}=\langle M ; F\rangle$ be a finite algebra and define $\mathscr{A}:=\mathbb{N} \mathbb{P}(\underline{\mathbf{M}})$. An alter ego of $\underline{\mathbf{M}}$ is a structured topological space $\underset{\sim}{\mathbf{M}}=\langle M ; G, H, R, \mathscr{T}\rangle$, on the same underlying set as $\underline{\mathbf{M}}$, such that:

(i) $G$ is a set of algebraic operations on $\underline{\mathbf{M}}$, that is, each $g \in G$ is a homomorphism $g: \underline{\mathbf{M}}^{n} \rightarrow \underline{\mathbf{M}}$, for some $n \in \mathbb{N} \cup\{0\}$;

(ii) $H$ is a set of algebraic partial operations on $\underline{\mathbf{M}}$, that is, each $h \in H$ is a homomorphism $h: \mathbf{D} \rightarrow \underline{\mathbf{M}}$, for some $n \in \mathbb{N}$ and $\mathbf{D}<\underline{\mathbf{M}}^{n}$;

(iii) $R$ is a set of algebraic relations on $\underline{\mathbf{M}}$, that is, each $r \in R$ is the underlying set of a subalgebra of $\underline{\mathbf{M}}^{n}$, for some $n \in \mathbb{N}$;

(iv) $\mathscr{T}$ is the discrete topology on $M$.


isomorphic copies of closed substructures of non-zero powers of $\mathbf{M}$. There is a pair of contravariant functors $\mathrm{D}: \mathscr{A} \rightarrow \mathscr{X}$ and $\mathrm{E}: \mathscr{X} \rightarrow \mathscr{A}$. For every $\mathbf{A} \in \mathscr{A}$, define $\mathrm{D}(\mathrm{A})$ to be the homset $\mathscr{A}(\mathbf{A}, \underline{\mathbf{M}})$ viewed as a closed substructure of $\mathbf{M}^{A}$. The structure $\mathrm{D}(\mathbf{A})$ is called the dual of $\mathbf{A}$, for each $\mathbf{A} \in \mathscr{A}$. For every $\mathbf{X} \in \mathscr{X}$, define $\mathrm{E}(\mathbf{X})$ to be the homset $\mathscr{X}(\mathbf{X}, \mathbf{M})$ viewed as a subalgebra of $\underline{\mathbf{M}}^{X}$. It remains to define $\mathrm{D}$ and $\mathrm{E}$ on morphisms. For $\varphi: \mathbf{A} \rightarrow \mathbf{B}$ in $\mathscr{A}$, define $\mathrm{D}(\varphi): \mathrm{D}(\mathbf{B}) \rightarrow \mathrm{D}(\mathbf{A})$ by $\mathrm{D}(\varphi)(x):=x \circ \varphi$, and for $\psi: \mathbf{X} \rightarrow \mathbf{Y}$ in $\mathscr{X}$, define $\mathrm{E}(\psi): \mathrm{E}(\mathbf{Y}) \rightarrow \mathrm{E}(\mathbf{X})$ by $\mathrm{E}(\psi)(\alpha):=\alpha \circ \psi$. 
For each $\mathbf{A} \in \mathscr{A}$, there is a natural embedding $e_{\mathbf{A}}: \mathbf{A} \hookrightarrow \mathrm{ED}(\mathbf{A})$, given by $e_{\mathbf{A}}(a)(x):=x(a)$, for all $a \in A$ and $x \in \mathscr{A}(\mathbf{A}, \underline{\mathbf{M}})$. Similarly, for each $\mathbf{X} \in \mathscr{X}$, we can define an embedding $\varepsilon_{\mathbf{X}}: \mathbf{X} \hookrightarrow \mathrm{DE}(\mathbf{X})$ by $\varepsilon_{\mathbf{X}}(x)(\alpha):=\alpha(x)$. If $e_{\mathrm{A}}$ is an isomorphism, for all $\mathbf{A} \in \mathscr{A}$, then we say that $\mathbf{M}$ yields a duality on $\mathscr{A}$. In this case, we have a representation for $\mathscr{A}$ : each algebra $\mathrm{A} \in \mathscr{A}$ is isomorphic to the algebra $\mathrm{ED}(\mathbf{A})$ of all morphisms from its dual $\mathrm{D}(\mathbf{A})$ into $\underset{\sim}{\mathbf{M}}$. If $e_{\mathbf{A}}$ and $\varepsilon_{\mathbf{X}}$ are isomorphisms, for all $\mathbf{A} \in \mathscr{A}$ and $\mathbf{X} \in \mathscr{X}$, then we say that $\mathbf{M}$ yields a full duality on $\mathscr{A}$. In this case, the categories $\mathscr{A}$ and $\mathscr{X}$ are dually equivalent. The algebra $\underline{\mathbf{M}}$ is (fully) dualisable if there is an alter ego of $\underline{\mathbf{M}}$ that yields a (full) duality on $\mathscr{A}$.

As we shall see in Section 5, full dualities are rather complicated. There is a simpler, stronger notion that we often use instead. First, let $S$ be a non-empty set and let $F_{\underline{M}}(S)$ denote the set of all $S$-ary term functions of $\underline{\mathbf{M}}$. A set $X \subseteq M^{S}$ is term closed if

$$
X=\bigcap\left\{\mathrm{eq}(\sigma, \tau) \mid \sigma, \tau \in F_{\underline{M}}(S) \text { and } \sigma \uparrow_{X}=\tau \uparrow_{X}\right\} .
$$

It is known that $\mathbf{M}$ yields a full duality on $\mathscr{A}$ if and only if $\mathbf{M}$ yields a duality on $\mathscr{A}$

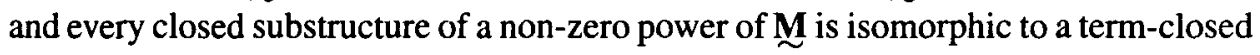
substructure of a power of $\underset{\sim}{\mathbf{M}}$ (see [1]). We now say that $\mathbf{M}$ yields a strong duality on $\mathscr{A}$ if $\mathbf{M}$ yields a duality on $\mathscr{A}$ and every closed substructure of a non-zero power of $\mathbf{M}$ is term closed. So every strong duality is also a full duality. At present, it is not known whether every full duality is also strong. The algebra $\underline{\mathbf{M}}$ is strongly dualisable if there is an alter ego of $\underline{\mathbf{M}}$ that yields a strong duality on $\mathscr{A}$.

By the First Strong Duality Theorem [1], the structure $\underset{\sim}{\mathbf{M}}$ yields a strong duality on $\mathscr{A}$ if and only if $\mathbf{M}$ yields a full duality on $\mathscr{A}$ and $\mathbf{M}$ is injective in $\mathscr{X}$. There are close connections between the injectivity of $\mathbf{M}$ in $\mathscr{X}$ and the injectivity of $\underline{\mathbf{M}}$ in $\mathscr{A}$ (see [1, Section 3.2]). The strong dualisability of an algebra seems to be related to how close the algebra is to being injective. Certainly, every dualisable algebra that is injective in the quasi-variety it generates is strongly dualisable. This follows from results of Willard [10], but it can also be proved directly using the results in [1].

LEMMA 1.1. Let $\underline{\mathbf{M}}$ be a finite algebra that is injective in $\mathbb{D S P}(\underline{\mathbf{M}})$. If $\underline{\mathbf{M}}$ is dualisable, then $\underline{\mathbf{M}}$ is strongly dualisable.

ProOF (Sketch). Assume that $\underline{\mathbf{M}}$ is dualisable. Then there is a relational structure $\underline{\mathbf{M}}=\langle M ; R, \mathscr{T}\rangle$ that yields a duality on $\mathscr{A}:=\mathbb{N} \mathbb{P}(\underline{\mathbf{M}})$. Define the set $G:=$ $\widetilde{U}\left\{\mathscr{A}\left(\underline{\mathbf{M}}^{n}, \underline{\mathbf{M}}\right) \mid n \in \mathbb{N} \cup\{0\}\right\}$ and define the alter ego $\underline{\mathbf{M}}^{\prime}:=\langle M ; G, R, \mathscr{T}\rangle$ of $\underline{\mathbf{M}}$. Then $\mathbf{M}^{\prime}$ also yields a duality on $\mathscr{A}$. Since $\underline{\mathbf{M}}$ is injective in $\mathscr{A}$, every algebraic partial operation on $\underline{\mathbf{M}}$ is the restriction of an algebraic operation on $\underline{\mathbf{M}}$. It now follows that $\mathbf{M}^{\prime}$ yields a strong duality on $\mathscr{A}$ by Exercise 3.1 and the Second Strong Duality Theorem, in [1]. (For the solution to Exercise 3.1, refer to the proof of Theorem 3.1.3 in [1].) 
In this paper, we make use of two general methods for showing that a dualisable algebra is strongly dualisable. The first method is due to Clark, Idziak, Sabourin, Szabó and Willard [2].

THEOREM 1.2 ([2, Lemma 4.8]). Let $\underline{\mathbf{M}}$ be a finite algebra and let $\underset{\sim}{\mathbf{M}}$ be an alter ego of $\underline{\mathbf{M}}$. Then $\underline{\mathbf{M}}$ yields a strong duality on $\mathscr{A}:=\square \triangle \mathbb{P}(\underline{\mathbf{M}})$ if and only if

(i) M yields a duality on $\mathscr{A}$, and

(ii) for every $\mathbf{A} \in \mathscr{A}$ and each proper closed substructure $\mathbf{X}$ of $\mathrm{D}(\mathbf{A})$, the maps in $X$ do not separate the elements of $A$.

The second method was introduced by Lampe, McNulty and Willard [8]. It is based on a result of Willard [10]. For a set $Y \subseteq \mathscr{A}\left(\underline{\mathbf{M}}^{n}, \underline{\mathbf{M}}\right)$, with $n \in \mathbb{N}$, define the natural product map $\sqcap Y: \underline{\mathbf{M}}^{n} \rightarrow \underline{\mathbf{M}}^{Y}$ by $\sqcap Y(a)(y):=y(a)$. We say that $\underline{\mathbf{M}}$ has enough algebraic operations if there is a map $f: \mathbb{N} \rightarrow \mathbb{N}$ for which the following condition holds:

for all $n \in \mathbb{N}$, all algebras $\mathbf{B} \leqslant \mathbf{A} \leqslant \underline{\mathbf{M}}^{n}$ and all homomorphisms $h: \mathbf{A} \rightarrow \underline{\mathbf{M}}$, there exists a set $Y \subseteq \mathscr{A}\left(\underline{\mathbf{M}}^{n}, \underline{\mathbf{M}}\right)$, with $|Y| \leqslant f(|B|)$, and a homomorphism $h^{\prime}: \Pi Y(\mathbf{A}) \rightarrow \underline{\mathbf{M}}$ such that the diagram below commutes.

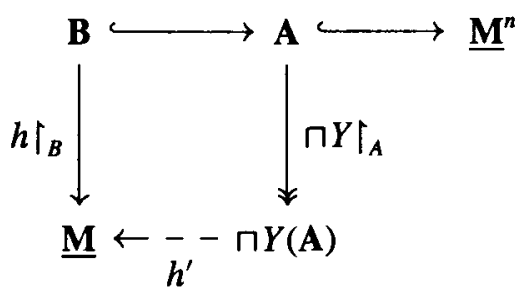

Although the definition of enough algebraic operations appears technical, it often provides a relatively easy way to lift dualisability to strong dualisability.

THEOREM 1.3 ([8, Theorem 4.3]). Let $\underline{\mathbf{M}}$ be a finite algebra with enough algebraic operations. If $\underline{\mathbf{M}}$ is dualisable, then $\underline{\mathbf{M}}$ is also strongly dualisable.

\section{Zero-kernel and one-kernel unary algebras are strongly dualisable}

The quasi-variety generated by a zero- or one-kernel unary algebra is especially simple. Consider a finite (not necessarily three-element) zero- or one-kernel unary algebra $\underline{\mathbf{M}}$ and define $\mathscr{A}:=\triangle \mathbb{S P}(\underline{\mathbf{M}})$. There is a finite set $\mathscr{B}$ of algebras that encapsulates the quasi-variety $\mathscr{A}$ : each finite algebra in $\mathscr{A}$ is 'nearly isomorphic' to a coproduct of algebras from $\mathscr{B}$. The finiteness of the quasi-variety $\mathscr{A}$ suggests that 
the algebra $\underline{\mathbf{M}}$ should be dualisable. This is the case, and it is proven in [4]. To show that $\underline{\mathbf{M}}$ is strongly dualisable, it now suffices to show that $\underline{\mathbf{M}}$ has enough algebraic operations. We begin by describing the finiteness of $\mathscr{A}$ precisely, using the definition of a gentle basis from [4].

Let $\mathbf{A}$ be a finite unary algebra. An element $a \in A$ is called an outer element of $\mathbf{A}$ if $\operatorname{sg}_{\mathrm{A}}(a)$ is a maximal one-generated subalgebra of $\mathbf{A}$. Otherwise, we say that $a \in A$ is an inner element of $\mathbf{A}$. Let $A_{\text {out }}$ denote the set of all outer elements of $\mathbf{A}$ and let $A_{\text {in }}$ denote the set of all inner elements of $\mathbf{A}$. Then $A_{\text {in }}$ is a subuniverse of $\mathbf{A}$. A surjection $\varphi: \mathbf{A} \rightarrow \mathbf{B}$ is said to be gentle if $\varphi \Gamma_{A_{\text {in }} \cup s_{\mathrm{A}_{A}(a)}}$ is one-to-one, for all $a \in A_{\text {out }}$.

Let $\underline{\mathbf{M}}$ be a finite unary algebra and define $\mathscr{A}:=\mathbb{D S P}(\underline{\mathrm{M}})$. An algebra $P$ is called a petal of $\mathscr{A}$ if $\mathbf{P}$ is a petal of an algebra belonging to $\mathscr{A}$. Let $\mathscr{B}$ be a set of finite petals of $\mathscr{A}$. Then $\mathscr{B}$ is a gentle basis for $\mathscr{A}$ if, for every finite petal $\mathbf{P}$ of $\mathscr{A}$, there is a gentle surjection $\varphi: \mathbf{P} \rightarrow \mathbf{B}$, for some $\mathbf{B} \in \mathscr{B}$. The following three results are proven in [4]. The first lemma shows that gentle surjections are 'nearly' isomorphisms.

LEMMA 2.1 ([4, Lemma 3.3]). Let $\mathbf{A}$ be a finite unary algebra and let $\varphi: \mathbf{A} \rightarrow \mathbf{B}$ be a gentle surjection. Then $\varphi$ is a retraction and, for each subalgebra $\mathbf{C}$ of $\mathbf{A}$ such that $\left.\varphi\right|_{C}$ is one-to-one, there is a coretraction $\psi: \mathbf{B} \hookrightarrow \mathbf{A}$ for $\varphi$ with $C \subseteq \psi(B)$.

LEMMA 2.2 ([4, Corollary 3.8]). Let $\underline{\mathbf{M}}$ be a finite unary algebra. Then $1 S \mathbb{P}(\underline{\mathrm{M}})$ has a finite gentle basis if and only if $\underline{\mathbf{M}}$ is a zero-kernel or one-kernel algebra.

THEOREM 2.3 ([4, Theorems 2.7 and 3.9]). Every finite zero-kernel or one-kernel unary algebra is dualisable.

We shall also use the following general lemma due to Hyndman [6].

LEMMA 2.4 ([6, Lemma 2.2]). Let $\underline{\mathbf{M}}$ be a finite algebra and define $\mathscr{A}:=0 \$ \mathbb{P}(\underline{\mathrm{M}})$. Let $\mathbf{B} \leqslant \underline{\mathbf{M}}^{n}$, for some $n \in \mathbb{N}$. Then there is a set $Z \subseteq \mathscr{A}\left(\underline{\mathbf{M}}^{n}, \underline{\mathbf{M}}\right)$ of projection functions such that $Z$ separates the elements of $B$ and $|Z| \leqslant|B|-1$.

Using the results above, we now prove that finite zero- and one-kernel algebras have enough algebraic operations.

LEMMA 2.5. Every finite zero-kernel or one-kernel unary algebra has enough algebraic operations.

ProOF. Let $\underline{\mathbf{M}}$ be a finite zero-kernel or one-kernel unary algebra. By Lemma 2.2, there is a finite gentle basis $\mathscr{B}$ for $\mathscr{A}:=\mathbb{D S P}(\mathbf{M})$. Choose some $k \in \mathbb{N}$ such that $k \geqslant|\mathscr{A}(\mathbf{P}, \underline{\mathbf{M}})|$, for all $\mathbf{P} \in \mathscr{B}$. Define the map $f: \mathbb{N} \rightarrow \mathbb{N}$ by $f(n):=k^{2} n+n-1$. Now let $n \in \mathbb{N}$, let $\mathbf{B} \leqslant \mathbf{A} \leqslant \underline{\mathbf{M}}^{n}$ and let $h: \mathbf{A} \rightarrow \underline{\mathbf{M}}$. 
Define $\mathscr{P}$ to be the set of all petals of $\underline{\mathbf{M}}^{n}$. Let $\mathscr{P}_{\mathrm{B}}$ denote the set of all petals $\mathbf{P}$ of $\underline{\mathbf{M}}^{n}$ such that $P \cap B \neq C_{\underline{M}^{n}}$. For each $\mathbf{P} \in \mathscr{P}$, there is a gentle surjection $\varphi_{\mathbf{P}}: \mathbf{P} \rightarrow \mathbf{P}^{*}$, where $\mathbf{P}^{*} \in \mathscr{B}$, and we can write $\mathscr{A}\left(\mathbf{P}^{*}, \underline{\mathbf{M}}\right)=\left\{x_{\mathbf{P} 1}, \ldots, x_{\mathbf{P} k}\right\}$. For each $\mathbf{P} \in \mathscr{P}$ and all $i, j \in\{1, \ldots, k\}$, we can define the algebraic operation $g_{\mathbf{P}_{i j}}: \underline{\mathbf{M}}^{n} \rightarrow \underline{\mathbf{M}}$ by

$$
g_{\mathbf{P} i j}:=\left(x_{\mathbf{P} i} \circ \varphi_{\mathbf{P}}\right) \sqcup \bigsqcup\left\{x_{\mathbf{Q} j} \circ \varphi_{\mathbf{Q}} \mid \mathbf{Q} \in \mathscr{P} \backslash\{\mathbf{P}\}\right\},
$$

as $\underline{\mathbf{M}}^{n}$ is the coproduct of its petals.

By Lemma 2.4 , there is a set $Z \subseteq \mathscr{A}\left(\underline{\mathbf{M}}^{n}, \underline{\mathbf{M})}\right.$ of projection functions such that $Z$ separates the elements of $B$ and $|Z| \leqslant|B|-1$. Define

$$
Y:=Z \cup\left\{g_{\mathbf{P}_{i j}} \mid \mathbf{P} \in \mathscr{P}_{\mathbf{B}} \text { and } i, j \in\{1, \ldots, k\}\right\} .
$$

Then $|Y| \leqslant|B|-1+k^{2}|B|=f(|B|)$. Define $\mu: \underline{\mathbf{M}}^{n} \rightarrow \underline{\mathbf{M}}^{Y}$ by $\mu:=\sqcap Y$. We wish to find a homomorphism $h^{\prime}: \mu(\mathbf{A}) \rightarrow \underline{\mathbf{M}}$ such that $h^{\prime} \circ \mu \uparrow_{B}=h \Gamma_{B}$. We can assume that the set $Y$ is not empty. (Otherwise, we have $|B|=1=|\mu(A)|$ and so we can define $h^{\prime}$ to have the same value as $h \Gamma_{B}$.)

The set $D:=\bigcup\left\{P \mid \mathbf{P} \in \mathscr{P} \backslash \mathscr{P}_{\mathbf{B}}\right\}$ determines a subalgebra $\mathbf{D}$ of $\underline{\mathbf{M}}^{n}$. We will show that $\mu(\mathbf{A})$ is the coproduct of $\left\{\mu(\mathbf{P} \cap \mathbf{A}) \mid \mathbf{P} \in \mathscr{P}_{\mathbf{B}}\right\} \cup\{\mu(\mathbf{D} \cap \mathbf{A})\}$ in $\mathscr{A}$. To do this, it suffices to prove that

$$
\left\{\mu(P \cap A) \backslash C_{\mu(\mathbf{A})} \mid \mathbf{P} \in \mathscr{P}_{\mathbf{B}}\right\} \cup\left\{\mu(D \cap A) \backslash C_{\mu(\mathbf{A})}\right\}
$$

is a partition of $\mu(A) \backslash C_{\mu(\mathrm{A})}$. Let $a \in(P \cap A) \backslash C_{\mathrm{A}}$ and let $b \in(Q \cap A) \backslash C_{\mathrm{A}}$, for some $\mathbf{P} \in \mathscr{P}_{\mathrm{B}}$ and $\mathbf{Q} \in \mathscr{P} \backslash\{\mathbf{P}\}$. It is now enough to show that $\mu(a) \neq \mu(b)$.

As $a \neq b$ in $M^{n}$, there is a homomorphism $z: \underline{\mathbf{M}}^{n} \rightarrow \underline{\mathbf{M}}$ such that $z(a) \neq z(b)$. By Lemma 2.1, there is a coretraction $\psi_{a}: \mathbf{P}^{*} \hookrightarrow \mathbf{P}$ for $\varphi_{\mathbf{P}}$ with $\operatorname{sg}_{\mathbf{P}}(a) \subseteq \psi_{a}\left(P^{*}\right)$. It follows that $\psi_{a} \circ \varphi_{\mathrm{P}}(a)=a$. The homomorphism $z \circ \psi_{a}: \mathbf{P}^{*} \rightarrow \underline{\mathbf{M}}$ belongs to $\mathscr{A}\left(\mathbf{P}^{*}, \underline{\mathbf{M}}\right)=\left\{x_{\mathbf{P} 1}, \ldots, x_{\mathbf{P}_{k}}\right\}$. So there is some $i \in\{1, \ldots, k\}$ such that $z \circ \psi_{a}=x_{\mathbf{P}}$ and therefore $x_{\mathbf{P}_{i}} \circ \varphi_{\mathrm{P}}(a)=z \circ \psi_{a} \circ \varphi_{\mathrm{P}}(a)=z(a)$. Similarly, there exists some $j \in\{1, \ldots, k\}$ with $x_{\mathbf{Q} j} \circ \varphi_{\mathbf{Q}}(b)=z(b)$. Thus

$$
g_{\mathbf{P} i j}(a)=x_{\mathbf{P} i} \circ \varphi_{\mathbf{P}}(a)=z(a) \neq z(b)=x_{\mathbf{Q} j} \circ \varphi_{\mathbf{Q}}(b)=g_{\mathbf{P}_{i j}}(b),
$$

whence $\mu(a) \neq \mu(b)$. We have shown that the algebra $\mu(\mathbf{A})$ is the coproduct of $\left\{\mu(\mathbf{P} \cap \mathbf{A}) \mid \mathbf{P} \in \mathscr{P}_{\mathbf{B}}\right\} \cup\{\mu(\mathbf{D} \cap \mathbf{A})\}$ in $\mathscr{A}$.

Now let $\mathbf{P} \in \mathscr{P}_{\mathbf{B}}$. To see that the surjection $\mu \uparrow_{P \cap A}: \mathbf{P} \cap \mathbf{A} \rightarrow \mu(\mathbf{P} \cap \mathbf{A})$ is gentle, let $a \in(P \cap A)_{\text {out }}$ and let $b, c \in(P \cap A)_{\text {in }} \cup \operatorname{sg}_{\mathrm{P} \cap A}(a)$ with $b \neq c$. As $(P \cap A)_{\text {in }} \subseteq P_{\text {in }}$ and $\varphi_{\mathbf{P}}$ is gentle, we have $\varphi_{\mathbf{P}}(b) \neq \varphi_{\mathbf{P}}(c)$. There must be some $i \in\{1, \ldots, k\}$ with $x_{\mathbf{P} i} \circ \varphi_{\mathbf{P}}(b) \neq x_{\mathbf{P} i} \circ \varphi_{\mathbf{P}}(c)$. So $g_{\mathbf{P} i 1}(b) \neq g_{\mathbf{P} i 1}(c)$ and therefore $\mu(b) \neq \mu(c)$. Thus $\mu\left\lceil_{P \cap A}: \mathbf{P} \cap \mathbf{A} \rightarrow \mu(\mathbf{P} \cap \mathbf{A})\right.$ is gentle. Since $Y$ separates the elements of $B$, the 
map $\mu \uparrow_{P \cap B}$ is one-to-one. It follows by, Lemma 2.1 , that there is a coretraction $\nu_{\mathbf{P}}: \mu(\mathbf{P} \cap \mathbf{A}) \hookrightarrow \mathbf{P} \cap \mathbf{A}$ for $\mu \uparrow_{P \cap A}$ such that $P \cap B \subseteq \nu_{\mathbf{P}} \circ \mu(P \cap A)$.

Since $\mu(\mathbf{A}) \leqslant \underline{\mathbf{M}}^{Y}$ and the set $Y$ is non-empty, there exists a homomorphism $x: \mu(\mathbf{A}) \rightarrow \underline{\mathbf{M}}$. We can now define the homomorphism $h^{\prime}: \mu(\mathbf{A}) \rightarrow \underline{\mathbf{M}}$ by

$$
h^{\prime}:=x \uparrow_{\mu(D \cap A)} \sqcup \bigsqcup\left\{h \circ v_{\mathbf{P}} \mid \mathbf{P} \in \mathscr{P}_{\mathbf{B}}\right\} .
$$

To see that $h^{\prime} \circ \mu \uparrow_{B}=h \uparrow_{B}$, let $\mathbf{P} \in \mathscr{P}_{\mathbf{B}}$ and let $b \in P \cap B$. Since $\nu_{\mathbf{P}}$ is a coretraction for $\mu \uparrow_{P \cap A}$ such that $P \cap B \subseteq \nu_{\mathrm{P}} \circ \mu(P \cap A)$, we have $\nu_{\mathrm{P}} \circ \mu \uparrow_{P \cap B}=\mathrm{id}_{P \cap B}$ and therefore

$$
h^{\prime} \circ \mu(b)=h \circ v_{\mathbf{P}} \circ \mu(b)=h(b) \text {. }
$$

Thus $\underline{\mathbf{M}}$ has enough algebraic operations.

The next theorem follows straight from Theorem 1.3, Theorem 2.3 and Lemma 2.5.

THEOREM 2.6. Every finite zero-kernel or one-kernel unary algebra is strongly dualisable.

We finish this section by considering quasi-injectivity.

LEMMA 2.7. Every finite zero-kernel or one-kernel unary algebra is n-quasi-injective, for some $n \in \mathbb{N}$.

ProOF. Let $\underline{\mathbf{M}}$ be a finite zero-kernel or one-kernel unary algebra. By Lemma 2.2, there is a finite gentle basis $\mathscr{B}$ for $\mathscr{A}:=\mathbb{U S P}(\underline{\mathrm{M}})$. Every algebra in $\mathscr{B}$ is a finite petal of $\mathscr{A}$. For each finite petal $\mathbf{P}$ of $\mathscr{A}$, define

$$
w_{\mathbf{P}}:=\max \left(\left\{d_{\mathbf{P}}(a, b) \mid a, b \in P \backslash C_{\mathbf{P}}\right\}\right) .
$$

Now define

$$
n:=\max \left(\left\{w_{\mathbf{B}} \mid \mathbf{B} \in \mathscr{B}\right\} \cup\{2\}\right) .
$$

Let $\mathbf{P}$ be a finite petal of $\mathscr{A}$. There is a gentle surjection $\varphi: \mathbf{P} \rightarrow \mathbf{B}$, for some $\mathbf{B} \in \mathscr{B}$. To see that $w_{\mathbf{P}} \leqslant n$, let $a, b \in P \backslash C_{\mathbf{P}}$ with $a \neq b$. Define the subuniverse $P_{a b}:=P_{\text {in }} \cup \operatorname{sg}_{\mathbf{P}}(\{a, b\})$ of $\mathbf{P}$.

Case (a): $\varphi \uparrow_{P_{a b}}$ is one-to-one. By Lemma 2.1 , there is a coretraction $\psi: \mathbf{B} \hookrightarrow \mathbf{P}$ for $\varphi$ with $a, b \in \psi(B)$. So $d_{\mathbf{P}}(a, b) \leqslant d_{\psi(\mathbf{B})}(a, b) \leqslant w_{\psi(\mathbf{B})}=w_{\mathbf{B}} \leqslant n$.

Case (b): $\left.\varphi\right|_{P_{a b}}$ is not one-to-one. Since $\varphi$ is gentle, we have $a, b \in P_{\text {out }}$ and $\operatorname{sg}_{\mathbf{P}}(a) \neq \operatorname{sg}_{\mathbf{P}}(b)$. There is $c_{a} \in \operatorname{sg}_{\mathbf{P}}(a) \backslash P_{\text {in }}$ and $c_{b} \in \operatorname{sg}_{\mathbf{P}}(b) \backslash P_{\text {in }}$ with $\varphi\left(c_{a}\right)=\varphi\left(c_{b}\right)$. We must have $\operatorname{sg}_{\mathbf{P}}(a)=\operatorname{sg}_{\mathbf{P}}\left(c_{a}\right)$ and $\operatorname{sg}_{\mathbf{P}}(b)=\operatorname{sg}_{\mathbf{P}}\left(c_{b}\right)$, since $c_{a}$ and $c_{b}$ are outer elements of $\mathbf{P}$. As $c_{a}$ and $c_{b}$ are connected by a fence in $\mathbf{P}$, there must be a unary 
term function $u$ of $\mathbf{A}$ such that $u\left(c_{a}\right) \in P_{\text {in }} \backslash C_{\mathbf{P}}$. This gives us $u\left(c_{a}\right)=u\left(c_{b}\right)$, since $\varphi\left(u\left(c_{a}\right)\right)=\varphi\left(u\left(c_{b}\right)\right)$ and $\varphi$ is gentle. Therefore $d_{\mathbf{P}}(a, b) \leqslant 2 \leqslant n$, as $a \in \operatorname{sg}_{\mathbf{P}}\left(c_{a}\right)$ and $b \in \operatorname{sg}_{\mathbf{P}}\left(c_{b}\right)$.

We have shown that $w_{\mathbf{P}} \leqslant n$, for all finite petals $\mathbf{P}$ of $\mathscr{A}$. To see that $\underline{\mathbf{M}}$ is $n$-quasiinjective, choose a pair of finite algebras $\mathbf{A}, \mathbf{B} \in \mathscr{A}$ with $\mathbf{B} \leqslant \mathbf{A}$. Let $x: \mathbf{B} \rightarrow \underline{\mathbf{M}}$ and assume that $x$ extends to $n_{\mathbf{A}}(B)$. Let $\mathbf{P}$ be a petal of $\mathbf{A}$ with $P \cap B \neq C_{\mathbf{A}}$. Then $P \subseteq n_{\mathbf{A}}(B)$, since $w_{\mathbf{P}} \leqslant n$. So $\left.x\right|_{P \cap B}$ extends to $\mathbf{P}$. Since $\mathbf{A}$ is the coproduct of its petals, it follows that $x$ extends to $\mathbf{A}$.

\section{Two-kernel three-element unary algebras that are strongly dualisable}

The family of two-kernel three-element unary algebras is surprisingly complicated. It contains strongly dualisable algebras, dualisable algebras that are not fully dualisable, and non-dualisable algebras. We begin this section by giving two different classifications of the two-kernel three-element unary algebras. To give the classifications, we require the following easy lemmas from [4].

LEMMA 3.1 ([4, Lemma 4.1]). Let M be a two-kernel three-element unary algebra. There is an isomorphic copy of $\underline{\mathbf{M}}$, on the set $\{0,1,2\}$, that has kernels $\{01 \mid 2\}$ and $\{02 \mid 1\}$.

LEMMA 3.2 ([4, Lemma 4.2]). Let $\underline{\mathbf{M}}$ be a two-kernel unary algebra, on the set $\{0,1,2\}$, with kernels $\{01 \mid 2\}$ and $\{02 \mid 1\}$. Then the unary term functions of $\underline{\mathbf{M}}$ all belong to the set $\{012,021\} \cup\{p p q, p q p \mid p, q \in M\}$.

We can now restrict our attention to those two-kernel algebras, on the set $\{0,1,2\}$, that have kernels $\{01 \mid 2\}$ and $\{02 \mid 1\}$. The most complicated algebra of this kind is $\underline{\mathbf{M}}^{\sharp}=\left\langle\{0,1,2\} ; F^{\sharp}\right\rangle$, where $F^{\sharp}:=\{012,021\} \cup\{p p q, p q p \mid p, q \in M\}$. Define the idempotent operations $f_{1}:=010$ and $f_{2}:=002$ in $F^{\sharp}$. The next result divides the two-kernel three-element unary algebras up into four types.

THEOREM 3.3 ([4, Theorem 4.3]). Let $\underline{\mathbf{M}}$ be a two-kernel unary algebra, on the set $\{0,1,2\}$, with kernels $\{01 \mid 2\}$ and $\{02 \mid 1\}$. Let $F$ be the set of unary term functions of $\underline{\mathrm{M}}$. Then at least one of the following is true:

$(2)_{\mathrm{O}} \quad$ each map in $F$ preserves the order $\preccurlyeq$ with $1 \preccurlyeq 0 \preccurlyeq 2$;

(2) $\left\{f_{1}, f_{2}\right\} \nsubseteq F$, and $\{p p q, p q p\} \subseteq F$, for some $p, q \in M$ with $p \neq q$;

$(2)_{\mathrm{M}} \quad\{010,001,110\} \subseteq F$ and $222 \notin F$, or $\{002,020,202\} \subseteq F$ and $111 \notin F$;

$(2)_{\mathrm{R}} \quad\left\{f_{1}, f_{2}\right\} \subseteq F$, and condition $(2)_{\mathrm{M}}$ fails. 
In [4], it is shown that the algebras of type $(2)_{O}$ and type $(2)_{R}$ are dualisable, and that the algebras of type (2) $)_{P}$ and type (2) ${ }_{M}$ are non-dualisable. We shall use a different classification for our characterisation of strong dualisability.

THEOREM 3.4. Let $\underline{\mathbf{M}}$ be a two-kernel unary algebra, on the set $\{0,1,2\}$, with kernels $\{01 \mid 2\}$ and $\{02 \mid 1\}$. Let $F$ be the set of unary term functions of $\underline{\mathbf{M}}$. Then at least one of the following is true:

(2) $\left\{f_{1}, f_{2}\right\} \nsubseteq F$, and $\{p p q, p q p\} \subseteq F$, for some $p, q \in M$ with $p \neq q$;

(2) Q $_{\mathrm{Q}}\{p p q, q p q\} \subseteq F$, for some $p, q \in M$ with $p \neq q$;

(2) $\mathrm{C} \quad\{101,220\} \subseteq F$;

(2) $\left\{f_{1}, f_{2}\right\} \subseteq F$, and conditions $(2)_{\mathrm{Q}}$ and $(2)_{\mathrm{C}}$ both fail.

PROOF. First assume that $\underline{\mathbf{M}}$ has type $(2)_{\mathrm{O}}$ but not type $(2)_{\mathrm{Q}}$. We will show that $\{010,002\} \subseteq F \subseteq\{012,010,002,000,111,222\}$. Since $\{01 \mid 2\}$ and $\{02 \mid 1\}$ are kernels of $\underline{M}$, there are $p, q, r, s \in M$, with $p \neq q$ and $r \neq s$, such that $p p q \in F$ and $r s r \in F$. As $\underline{\mathrm{M}}$ does not have type (2) $)_{\mathrm{Q}}$, we must have $q p q \notin F$ and $s s r \notin F$. The only non-constant maps in $M^{M}$ that preserve the order $\preccurlyeq$ are $012,110,112,002,010,212$ and 202. As rsro110 = rsro112=ssr $\notin F$ and $p p q \circ 212=p p q \circ 202=q p q \notin F$, it follows that $\{010,002\} \subseteq F \subseteq\{012,010,002,000,111,222\}$.

To see that the four types in the statement of the theorem are exhaustive, assume that $\underline{\mathrm{M}}$ has neither type (2) $)_{\mathrm{P}}$, type (2) Q $_{\mathrm{Q}}$, nor type (2) $\mathrm{C}$. We need to prove that $\left\{f_{1}, f_{2}\right\} \subseteq F$. Since $\underline{\mathbf{M}}$ does not have type $(2)_{Q}$, we have $\{110,010\} \nsubseteq F$ and $\{202,002\} \nsubseteq F$. Therefore $\underline{\mathbf{M}}$ does not have type $(2)_{\mathrm{M}}$. So, by Theorem 3.3, we can assume that $\underline{\mathbf{M}}$ has type (2) . We have just shown that this implies that $\left\{f_{1}, f_{2}\right\} \subseteq F$.

Throughout the rest of this paper, we shall prove that the algebras of type (2)s are strongly dualisable, and that the algebras of type $(2)_{Q}$ and type $(2)_{C}$ are not fully dualisable. This will provide us with plenty of examples of dualisable algebras that are not fully dualisable: for instance, each algebra $\langle\{0,1,2\} ; F\rangle$ such that $\{101,220\} \subseteq$ $F \subseteq F^{\sharp}$.

In [3] (see also [4]), it is shown that a finite unary algebra is dualisable if its operations form a set of lattice endomorphisms. The proof is particularly short and elegant. So it is slightly surprising that not all of these lattice-endomorphism unary algebras are fully dualisable. In fact, most of the two-kernel three-element latticeendomorphism unary algebras are not fully dualisable. In the proof of Theorem 3.4, we showed that a two-kernel unary algebra whose operations preserve the order $\preccurlyeq$, with $1 \preccurlyeq 0 \preccurlyeq 2$, must have type (2) $\mathrm{Q}$ unless it is polynomially isomorphic to $\langle\{0,1,2\} ; 010,002\rangle$.

Every algebra of type $(2)_{S}$ also has type $(2)_{R}$, and is therefore dualisable. We shall prove that every algebra of type $(2)_{S}$ has enough algebraic operations and is therefore 
strongly dualisable. The following lemma has nearly the same proof as that of Lemma 4.7 in [4].

LeMMA 3.5. Assume that $\underline{\mathbf{M}}$ has type (2)s. Let $\mathbf{A} \in \mathbb{D S P}(\underline{\mathbf{M}})$, let $\mathbf{A}_{*} \leqslant \mathbf{A}$ such that $f_{1}(A) \cup f_{2}(A) \subseteq A_{*}$ and let $x: \mathbf{A}_{*} \rightarrow \underline{\mathbf{M}}$ be a homomorphism. Then $x$ has an extension to $\mathbf{A}$ if and only if $x\left(f_{1}(a)\right)=0$ or $x\left(f_{2}(a)\right)=0$, for all $a \in A \backslash A_{*}$. Moreover, if $x$ has an extension to $\mathbf{A}$, then that extension is unique.

Assume that $\underline{\mathbf{M}}$ has type (2) $\mathrm{s}$ and let $F$ be the set of unary term functions of $\underline{\mathbf{M}}$. Then $\{101,220\} \nsubseteq F$, since $\underline{\mathbf{M}}$ does not have type $(2)_{\mathrm{C}}$. We shall consider the two cases $101,220 \notin F$ and $101 \in F$ separately. The case $220 \in F$ is symmetric, under conjugation by 021 , to the case $101 \in F$. To see this, assume that $220 \in F$. We can create an isomorphic copy of $\underline{\mathbf{M}}$ by interchanging the labels 1 and 2. More precisely, there is a unary algebra $\underline{\mathbf{M}}^{\prime}$, on the set $\{0,1,2\}$, such that $021: \underline{\mathbf{M}} \rightarrow \underline{\mathbf{M}}^{\prime}$ is an isomorphism. The set $F^{\prime}:=\{021 \circ u \circ 021 \mid u \in F\}$ is the set of all unary term functions of $\underline{\mathbf{M}}^{\prime}$. It is easy to check that $\underline{\mathbf{M}}^{\prime}$ has type (2) and that $101 \in F^{\prime}$.

LEMMA 3.6. Let $\underline{\mathrm{M}}$ be a unary algebra with type (2) $\mathrm{S}$.

(i) If neither 101 nor 220 is a term function of $\underline{\mathbf{M}}$, then all the unary term functions of $\underline{\mathbf{M}}$ belong to $\{012,021,010,020,001,002,000,111,222\}$.

(ii) If 101 is a term function of $\underline{\mathbf{M}}$, then all the unary term functions of $\underline{\mathbf{M}}$ belong to $\{012,101,010,002,000,111,222\}$.

Proof. Let $F$ denote the set of all unary term functions of $\underline{\mathbf{M}}$ and assume that $220 \notin F$. Since $010,002 \in F$ and $\underline{\mathbf{M}}$ does not have type $(2)_{\mathrm{Q}}$, we know that $110 \notin F$ and $202 \notin F$. Let $p p q \in F$ with $p \neq q$. Then $010 \circ p p q \in F$ and $002 \circ p p q \in F$. As $110,220 \notin F$, this implies that $p=0$. We have $212 \notin F$, as $002 \circ 212=202 \notin F$. Since $F \subseteq F^{\sharp}$, by Lemma 3.2, it now follows that

$$
F \subseteq\{012,021,010,020,101,121,001,002,000,111,222\} .
$$

To prove (i), assume that $101 \notin F$. Then $121 \notin F$, as $010 \circ 121=101 \notin F$. So claim (i) holds. To prove (ii), assume that $101 \in F$. We must have $021,001 \notin F$, since $101 \circ 021=101 \circ 001=110 \notin F$. As $020 \circ 101=202 \notin F$ and $121 \circ 101=212 \notin F$, we have $020,121 \notin F$. Thus claim (ii) holds.

Given a set $S$, for each $m \in M$ we use $\widehat{m}$ to denote the constant map in $M^{S}$ with value $m$.

LEMMA 3.7. Let $\underline{\mathbf{M}}$ be a unary algebra with type (2)s. Assume that neither 101 nor 220 is a term function of $\underline{\mathbf{M}}$. Let $\mathbf{B} \leqslant \mathbf{A}$ in $\triangle \mathbb{S P}(\underline{\mathbf{M}})$ and let $x: \mathbf{B} \rightarrow \underline{\mathbf{M}}$. Then the following are equivalent: 
(i) $x$ extends to $\mathbf{A}$;

(ii) $x$ extends to $\mathbf{1}_{\mathbf{A}}(B)$;

(iii) whenever $u_{1} \in\{010,020\}$ and $u_{2} \in\{002,001\}$ are term functions of $\underline{\mathbf{M}}$ and $a \in A$ with $u_{1}(a), u_{2}(a) \in B$, we have $x\left(u_{1}(a)\right)=0$ or $x\left(u_{2}(a)\right)=0$.

In particular, the algebra $\underline{\mathrm{M}}$ is 1-quasi-injective.

PROOF. Define $F$ to be the set of all unary term functions of $\underline{\mathbf{M}}$. We can assume that $\mathbf{A} \leqslant \underline{\mathbf{M}}^{S}$, for some set $S$. Clearly (i) implies (ii). To see that (ii) implies (iii), assume that $\bar{x}: \mathbf{1}_{\mathbf{A}}(B) \rightarrow \underline{\mathbf{M}}$ is an extension of $x$. Let $a \in A$ and let $u_{1} \in F \cap\{010,020\}$ and $u_{2} \in F \cap\{002,001\}$, with $u_{1}(a), u_{2}(a) \in B$, such that $x\left(u_{1}(a)\right) \neq 0$. We want to show that $x\left(u_{2}(a)\right)=0$. First assume that $u_{1}(a) \in C_{\mathrm{A}} \subseteq\{\widehat{0}, \widehat{1}, \widehat{2}\}$. Then $u_{1}(a) \neq \widehat{0}$, and so $a=\widehat{1}$. Since $000=f_{1} \circ f_{2}$ is a constant term function of $\underline{\mathbf{M}}$, this implies that $x\left(u_{2}(a)\right)=x(\widehat{0})=0$. Now assume that $u_{1}(a) \notin C_{\mathrm{A}}$. Then $a \in 1_{\mathrm{A}}(B)$ and $u_{1}(\bar{x}(a))=x\left(u_{1}(a)\right) \neq 0$. So $\bar{x}(a)=1$ and therefore $x\left(u_{2}(a)\right)=u_{2}(\bar{x}(a))=0$.

It remains to show that (iii) implies (i). So assume that condition (iii) holds. By Lemma 3.6 (i), the set $A_{*}:=A \cap\left(\{0,1\}^{S} \cup\{0,2\}^{S}\right)$ determines a subalgebra of $\mathbf{A}$. We want to define $x_{*}: \mathbf{A}_{*} \rightarrow \underline{\mathbf{M}}$ by

$$
x_{*}(a)= \begin{cases}2 & \text { if } u(a) \in x^{-1}(u(2)), \text { for some } u \in F \cap\{002,001\} \\ 1 & \text { if } u(a) \in x^{-1}(u(1)), \text { for some } u \in F \cap\{010,020\} \\ 0 & \text { otherwise. }\end{cases}
$$

To see that $x_{*}$ is well defined, let $u_{1} \in F \cap\{010,020\}, u_{2} \in F \cap\{002,001\}$ and $a \in A_{*}$ with $u_{1}(a), u_{2}(a) \in B$. Then $x\left(u_{1}(a)\right)=0 \neq u_{1}(1)$ or $x\left(u_{2}(a)\right)=0 \neq u_{2}(2)$, by (iii). So $x_{*}$ is well defined.

Now let $b \in B \cap A_{*}$. Then $x_{*}(b)=2$ implies $x(b)=2$, and

$$
x(b)=2 \Longrightarrow 002(x(b))=2 \Longrightarrow x(002(b))=002(2) \Longrightarrow x_{*}(b)=2 .
$$

Similarly, we have $x_{*}(b)=1$ if and only if $x(b)=1$. Thus $x_{*}$ extends $x \uparrow_{B \cap A_{*}}$. Using Lemma 3.6 (i), it is easy to check that $x_{*}$ is a homomorphism.

We shall prove that $x_{*}$ extends to a homomorphism $\bar{x}: \mathbf{A} \rightarrow \underline{\mathbf{M}}$ using Lemma 3.5. Choose some $a \in A \backslash A_{*}$ and suppose that $x_{*}\left(f_{1}(a)\right) \neq 0$ and $x_{*}\left(f_{2}(a)\right) \neq 0$. Since $f_{1}$ and $f_{2}$ are both idempotent, we must have $x_{*}\left(f_{1}(a)\right)=1$ and $x_{*}\left(f_{2}(a)\right)=2$. There exist $u_{1} \in F \cap\{010,020\}$ and $u_{2} \in F \cap\{002,001\}$ such that $u_{1} \circ f_{1}(a) \in x^{-1}\left(u_{1}(1)\right)$ and $u_{2} \circ f_{2}(a) \in x^{-1}\left(u_{2}(2)\right)$. This implies that $x\left(u_{1} \circ f_{1}(a)\right) \neq 0$ and $x\left(u_{2} \circ f_{2}(a)\right) \neq 0$, which contradicts (iii). So there is an extension $\bar{x}: \mathbf{A} \rightarrow \underline{\mathbf{M}}$ of $x_{*}$. By Lemma 3.5, the extension $x: \mathbf{B} \rightarrow \underline{\mathbf{M}}$ of $x \uparrow_{B \cap A_{*}}$ is unique. Thus $\bar{x}$ is an extension of $x$, as $\bar{x} \uparrow_{B \cap A_{*}}=x \uparrow_{B \cap A_{*}}$.

THEOREM 3.8. Let $\underline{\mathbf{M}}$ be a unary algebra, with type (2)s, such that neither 101 nor 220 is a term function of $\underline{\mathbf{M}}$. Then $\underline{\mathbf{M}}$ is strongly dualisable. 
Proof. By [4], the algebra $\underline{\mathbf{M}}$ is dualisable. So, using Theorem 1.3, it will follow that $\underline{\mathbf{M}}$ is strongly dualisable once we have shown that $\underline{\mathbf{M}}$ has enough algebraic operations. Define $f: \mathbb{N} \rightarrow \mathbb{N}$ by $f(n):=n$. Let $n \in \mathbb{N}$, let $\mathbf{B} \leqslant \mathbf{A} \leqslant \underline{\mathbf{M}}^{n}$ and let $h: \mathbf{A} \rightarrow \underline{\mathbf{M}}$.

By Lemma $3.6(\mathrm{i})$, the set $f_{1}\left(M^{n}\right) \cup f_{2}\left(M^{n}\right)=\{0,1\}^{n} \cup\{0,2\}^{n}$ determines a subalgebra of $\underline{\mathbf{M}}^{n}$. Let $m \in\{1,2\}$ and let $b \in f_{m}(B) \backslash\{\widehat{0}\}$. Using Lemma 3.5 and Lemma 3.6 (i), there is a homomorphism $g_{b}: \underline{M}^{n} \rightarrow \underline{M}$ such that, for all $a \in f_{1}\left(M^{n}\right) \cup f_{2}\left(M^{n}\right)$, we have

$$
g_{b}(a)= \begin{cases}m & \text { if } a=b \text { or } a=\widehat{m} ; \\ 021(m) & \text { if } a=021(b) \text { or } a=021(\widehat{m}) ; \\ 0 & \text { otherwise. }\end{cases}
$$

Define

$$
Y:=\left\{g_{b} \mid b \in\left(f_{1}(B) \cup f_{2}(B)\right) \backslash\{\hat{0}\}\right\} .
$$

Then $|Y| \leqslant|B|-1 \leqslant f(|B|)$.

Define the homomorphism $\mu: \underline{\mathbf{M}}^{n} \rightarrow \underline{\mathbf{M}}^{Y}$ by $\mu:=\Pi Y$. To see that $\left.\mu\right|_{B}$ is an embedding, we need to show that $Y$ separates the elements of $B$. Let $b, c \in B$ with $b \neq c$. Then $f_{m}(b) \neq f_{m}(c)$, for some $m \in\{1,2\}$. We can assume that $f_{m}(b) \neq \widehat{0}$ and $f_{m}(c) \neq \widehat{m}$. So $g_{f_{m}(b)}\left(f_{m}(b)\right)=m \neq g_{f_{m}(b)}\left(f_{m}(c)\right)$, which implies that $\mu\left(f_{m}(b)\right) \neq \mu\left(f_{m}(c)\right)$ and therefore $\mu(b) \neq \mu(c)$.

We shall use Lemma 3.7 to prove that $h \circ\left(\mu \Gamma_{B}\right)^{-1}: \mu(\mathbf{B}) \rightarrow \underline{\mathbf{M}}$ extends to $\mu(\mathbf{A})$. Choose any $a \in A$. Let $u_{1}$ and $u_{2}$ be unary term functions of $\underline{\mathbf{M}}$, with $u_{1} \in\{010,020\}$ and $u_{2} \in\{002,001\}$, such that $u_{1}(\mu(a)), u_{2}(\mu(a)) \in \mu(B)$. Define $m_{1}:=u_{1}(1)$ and $m_{2}:=u_{2}(2)$. Then $f_{m_{1}} \circ u_{1}=u_{1}$ and $f_{m_{2}} \circ u_{2}=u_{2}$. So there is some $b_{1} \in f_{m_{1}}(B)$ and $b_{2} \in f_{m_{2}}(B)$ with $\mu\left(u_{1}(a)\right)=\mu\left(b_{1}\right)$ and $\mu\left(u_{2}(a)\right)=\mu\left(b_{2}\right)$. We want to show that $h\left(b_{1}\right)=0$ or $h\left(b_{2}\right)=0$. So we can assume that $b_{1}, b_{2} \neq \widehat{0}$. Since $\mu \uparrow_{B}$ is one-to-one, we have $\mu\left(b_{1}\right), \mu\left(b_{2}\right) \neq \mu(\widehat{0})$. This implies that $a \notin\{\widehat{1}, \widehat{2}\}$, and so $u_{1}(a), u_{2}(a) \notin\{\widehat{1}, \widehat{2}\}$. For each $i \in\{1,2\}$, we have $m_{i}=g_{b_{i}}\left(b_{i}\right)=g_{b_{i}}\left(u_{i}(a)\right)$ and therefore $u_{i}(a)=b_{i}$. As $h \Gamma_{B}$ extends to A, it follows, by Lemma 3.7, that $h\left(b_{1}\right)=h\left(u_{1}(a)\right)=0$ or $h\left(b_{2}\right)=h\left(u_{2}(a)\right)=0$. We have shown that

$$
h \circ\left(\mu \Gamma_{B}\right)^{-1}\left(u_{j}(\mu(a))\right)=h \circ\left(\mu \Gamma_{B}\right)^{-1}\left(\mu\left(b_{j}\right)\right)=h\left(b_{j}\right)=0,
$$

for some $j \in\{1,2\}$. Hence $h \circ\left(\left.\mu\right|_{B}\right)^{-1}$ extends to $\mu(\mathbf{A})$, whence $\underline{\mathbf{M}}$ has enough algebraic operations.

To make the next two proofs easier to read, we introduce some notation. Assume that $\underline{\mathbf{M}}$ has type (2) and let $\mathbf{A} \in \mathbb{D S P}(\underline{\mathbf{M}})$. There is a natural binary relation $\frown_{\mathbf{A}}$ on $A$ that reflects part of the structure of A. For all $a, b \in A$, we set $a \frown_{A} b$ if and only if there is some $c \in A$ such that $a=f_{1}(c)$ and $b=f_{2}(c)$. 
LEMMA 3.9. Let $\underline{\mathrm{M}}$ be a unary algebra with type (2) $\mathrm{s}$. Assume that 101 is a term function of $\underline{\mathbf{M}}$. Let $\mathbf{B} \leqslant \mathbf{A}$ in $\triangle \mathbb{S P}(\underline{\mathbf{M}})$ and let $x: \mathbf{B} \rightarrow \underline{\mathbf{M}}$. Then the following are equivalent:

(i) $x$ extends to $\mathbf{A}$;

(ii) $x$ extends to $\mathbf{1}_{\mathbf{A}}(B)$;

(iii) the following conditions both hold:

(1) $x(b)=0$ or $x(c)=0$, for all $b, c \in B$ such that $b \frown_{A} c$;

(2) $x(b)=0$ or $x(c)=0$, for all $b, c \in B$ and $a \in A$ such that $a \frown_{A} b$ and $101(a) \frown \mathrm{A} c$.

In particular, the algebra $\underline{\mathrm{M}}$ is 1-quasi-injective.

Proof. Assume that $\mathbf{A} \leqslant \underline{\mathbf{M}}^{S}$, for some set $S$. To prove that (ii) implies (iii), assume that $x$ extends to a homomorphism $\bar{x}: \mathbf{1}_{\mathbf{A}}(B) \rightarrow \underline{\mathbf{M}}$. We first prove two claims.

$(*)_{1}$ Let $a, b \in A$, with $a \in C_{\mathrm{A}}$ or $b \in C_{\mathrm{A}}$, such that $a \frown_{\mathrm{A}} b$. Then $a=\widehat{0}$ or $b=\widehat{0}$.

There is some $c \in A$ with $f_{1}(c)=a$ and $f_{2}(c)=b$. Since either $a$ or $b$ belongs to the set $\{\widehat{0}, \widehat{1}, \widehat{2}\}$, we must have $c \in\{0, m\}^{S}$, for some $m \in\{1,2\}$. So $a=f_{1}(c)=\widehat{0}$ or $b=f_{2}(c)=\widehat{0}$.

$(*)_{2}$ Let $a \in A$ and $b \in B \backslash C_{\mathrm{A}}$ such that $a \frown_{\mathrm{A}} b$. Then $\bar{x}(a)=0$ or $x(b)=0$.

There is some $c \in A$ such that $f_{1}(c)=a$ and $f_{2}(c)=b$. Since $b \notin C_{\mathrm{A}}$, we have $a, c \in 1_{\mathbf{A}}(B)$. Assume that $x(b) \neq 0$. Then $f_{2}(\bar{x}(c))=x(b) \neq 0$ and so $\bar{x}(c)=2$. Therefore $\bar{x}(a)=\bar{x}\left(f_{1}(c)\right)=f_{1}(\bar{x}(c))=0$.

We can now show that (iii) holds. Since 000 is a term function of $\underline{\underline{M}}$, we have $x(\widehat{0})=0$. So (1) follows straight from claims $(*)_{1}$ and $(*)_{2}$. To see that $(2)$ holds, let $b, c \in B$ and $a \in A$, with $a \frown_{\mathrm{A}} b$ and $101(a) \frown_{\mathrm{A}} c$, such that $x(b) \neq 0$. First assume that $b, c \notin C_{\mathrm{A}}$. By $(*)_{2}$, we must have $\bar{x}(a)=0$ and therefore $\bar{x}(101(a))=$ $101(\bar{x}(a))=1$. Using $(*)_{2}$ again, we get $x(c)=0$. Now assume that $b \in C_{\mathrm{A}}$. Then $b \neq \widehat{0}$, since $x(b) \neq 0$. So $a=\widehat{0}$, by $(*)_{1}$, and therefore $101(a)=\widehat{1}$. As 101 $(a) \frown_{\mathrm{A}} c$, it follows that $c=\widehat{0}$, whence $x(c)=0$. Finally, assume that $c \in C_{\mathrm{A}}$. Since $b \neq \widehat{0}$ and $a \frown_{A} b$, we have $a \neq \widehat{1}$. This implies that $101(a) \neq \widehat{0}$, whence $c=\widehat{0}$, by $(*)_{1}$. Thus (iii) is satisfied.

To prove that (iii) implies (i), assume that (iii) holds. By Lemma 3.6 (ii), the sets $A_{1}:=f_{1}(A) \cup C_{\mathrm{A}}$ and $A_{2}:=f_{2}(A) \cup C_{\mathrm{A}}$ both determine subalgebras of $\mathrm{A}$. Let $\mathscr{T}$ be a transversal of $\left\{\{a, 101(a)\} \mid a \in f_{1}(A)\right\}$ and define the homomorphism $x_{1}: \mathbf{A}_{1} \rightarrow \underline{\mathbf{M}}$ such that, for all $a \in \mathscr{T}$, we have

$$
x_{1}(a)= \begin{cases}x(a) & \text { if } a \in B \\ 0 & \text { if } a \notin B \text { and } a \frown_{A} b, \text { for some } b \in x^{-1}(2) \\ 1 & \text { otherwise. }\end{cases}
$$


We can define $x_{2}: \mathbf{A}_{2} \rightarrow \underline{\mathbf{M}}$ by

$$
x_{2}(a)= \begin{cases}x(a) & \text { if } a \in B \\ 0 & \text { otherwise }\end{cases}
$$

Now define $A_{*}:=A_{1} \cup A_{2}$ and $x_{*}:=x_{1} \cup x_{2}: \mathbf{A}_{*} \rightarrow \underline{\mathbf{M}}$.

To see that $x_{*}$ extends to $\mathbf{A}$, using Lemma 3.5, let $a \in A \backslash A_{*}$ with $x_{*}\left(f_{2}(a)\right)=2$. Then $f_{2}(a) \in x^{-1}(2)$. First assume that $f_{1}(a) \in B$. Then $x_{*}\left(f_{1}(a)\right)=x\left(f_{1}(a)\right)=0$, by (1). Now assume that $f_{1}(a) \notin B$ and $f_{1}(a) \in \mathscr{T}$. Then $x_{*}\left(f_{1}(a)\right)=x_{1}\left(f_{1}(a)\right)=0$. Finally, assume that $f_{1}(a) \notin B$ and $101(a) \in \mathscr{T}$. We must have $x_{*}(101(a))=$ $x_{1}(101(a))=1$, by $(2)$, and therefore $x_{*}\left(f_{1}(a)\right)=101\left(x_{*}(101(a))\right)=0$. So there is a homomorphism $\bar{x}: \mathbf{A} \rightarrow \underline{\mathbf{M}}$ that extends $x_{*}$. By Lemma 3.5, the extension $x: \mathbf{B} \rightarrow \underline{\mathbf{M}}$ of $\left.x\right|_{B \cap A_{*}}=\left.\bar{x}\right|_{B \cap A_{*}}$ is unique, whence $\bar{x}$ extends $x$.

THEOREM 3.10. Let $\underline{\mathbf{M}}$ be a unary algebra, with type (2), such that either 101 or 220 is a term function of $\underline{\mathbf{M}}$. Then $\underline{\mathbf{M}}$ is strongly dualisable.

PROOF. By symmetry, we can assume that 101 is a term function of $\underline{\mathbf{M}}$. The algebra $\underline{\mathbf{M}}$ is dualisable, by [4]. So, using Theorem 1.3, it suffices to show that $\underline{\mathbf{M}}$ has enough algebraic operations. Define $\mathscr{A}:=\mathbb{N S P}(\underline{\mathbf{M}})$ and define the map $f: \mathbb{N} \rightarrow \mathbb{N}$ by $f(n):=3 n-2$. Let $n \in \mathbb{N}$, let $\mathbf{B} \leqslant \mathbf{A} \leqslant \underline{\mathbf{M}}^{n}$ and let $h: \mathbf{A} \rightarrow \underline{\mathbf{M}}$.

Now let $b \in f_{2}(B) \backslash\{\widehat{0}\}$. By Lemma 3.6 (ii), the set $f_{2}\left(M^{n}\right) \cup C_{\underline{M}^{n}}$ determines a subalgebra of $\underline{\mathrm{M}}^{n}$. Using Lemma 3.6 (ii) and Lemma 3.9, there is a homomorphism $g_{b}: \underline{\mathbf{M}}^{n} \rightarrow \underline{\mathbf{M}}$ such that, for all $a \in f_{2}\left(M^{n}\right)$, we have

$$
g_{b}(a)= \begin{cases}2 & \text { if } a=b \text { or } a=\widehat{2} \\ 0 & \text { otherwise. }\end{cases}
$$

The set $f_{1}\left(M^{n}\right) \cup C_{\underline{M}^{n}}$ determines a subalgebra of $\underline{\mathbf{M}}^{n}$. By Lemma 3.6 (ii) and Lemma 3.9, there is a homomorphism $g_{b}^{\prime}: \underline{\mathbf{M}}^{n} \rightarrow \underline{\mathbf{M}}$ such that, for $a \in f_{1}\left(M^{n}\right)$, we have

$$
g_{b}^{\prime}(a)= \begin{cases}g_{b}(a) & \text { if } a \frown_{\mathrm{A}} b \text { or } 101(a) \frown_{\mathrm{A}} b \\ 101\left(g_{b}(a)\right) & \text { otherwise. }\end{cases}
$$

By Lemma 2.4 , there is a set $Z \subseteq \mathscr{A}\left(\underline{\mathbf{M}}^{n}, \underline{\mathbf{M}}\right)$ of projections such that $Z$ separates the elements of $B$ and $|Z| \leqslant|B|-1$. Define

$$
Y:=Z \cup\left\{g_{b}, g_{b}^{\prime} \mid b \in f_{2}(B) \backslash\{\widehat{0}\}\right\} .
$$

Then $|Y| \leqslant|B|-1+2(|B|-1) \leqslant f(|B|)$.

Define $\mu: \underline{\mathbf{M}}^{n} \rightarrow \underline{\mathbf{M}}^{Y}$ by $\mu:=\sqcap Y$. Then $\mu \uparrow_{B}$ is an embedding, as $Y$ separates the elements of $B$. We shall begin by proving the following claim. 
(*) Let $a \in A$ and $b \in B$ with $\mu(a) \frown_{\mu(\mathbf{A})} \mu(b)$. Then $f_{1}(a) \frown_{\mathrm{A}} b$.

Since $f_{1}(a) \frown \widehat{0}$, we can assume that $b \in B \backslash\{\widehat{0}\}$. There is $c \in A$ with $f_{1}(\mu(c))=$ $\mu(a)$ and $f_{2}(\mu(c))=\mu(b)$. As $f_{1}$ is idempotent, this implies that $\mu\left(f_{1}(c)\right)=$ $\mu\left(f_{1}(a)\right)$. We have $\mu\left(f_{2}(b)\right)=\mu(b)$ and therefore $b \in f_{2}(B)$, as $\mu \digamma_{B}$ is one-to-one. This gives us $g_{b}\left(f_{2}(c)\right)=g_{b}(b)=2$, which implies that $g_{b}(c)=2$, and also that $f_{2}(c)=b$ or $c=\widehat{2}$. So $g_{b}^{\prime}\left(f_{1}(a)\right)=g_{b}^{\prime}\left(f_{1}(c)\right)=g_{b}\left(f_{1}(c)\right)=g_{b}\left(f_{1}(a)\right)$, which tells us that $f_{1}(a) \frown_{\mathbf{A}} b$ or $101(a) \frown_{\mathbf{A}} b$. We have $g_{b}(b)=2$ and

$$
g_{b}(101(a))=101\left(g_{b}\left(f_{1}(a)\right)\right)=101\left(g_{b}\left(f_{1}(c)\right)\right)=101\left(g_{b}(c)\right)=101(2)=1 .
$$

As $g_{b}$ preserves $f_{1}$ and $f_{2}$, it follows that $101(a){\digamma_{\mathrm{A}}} b$. Thus $f_{1}(a) \frown_{\mathrm{A}} b$, and (*) holds.

We will use Lemma 3.9 to prove that $h \circ\left(\mu \uparrow_{B}\right)^{-1}: \mu(\mathbf{B}) \rightarrow \underline{\mathbf{M}}$ extends to $\mu(\mathbf{A})$. To see that (1) holds, let $b, c \in B$ such that $\mu(b) \frown_{\mu(A)} \mu(c)$. Then $f_{1}(b) \frown_{A} c$, by (*). We have $f_{1}(b)=b$, as $f_{1}(\mu(b))=\mu(b)$ and $\mu \Gamma_{B}$ is one-to-one. So $h(b)=0$ or $h(c)=0$, by Lemma 3.9, since $h \uparrow_{B}$ extends to A.

To check that (2) holds, let $b, c \in B$ and $a \in A$ such that $\mu(a) \frown_{\mu(A)} \mu(b)$ and $101(\mu(a)) \frown_{\mu(A)} \mu(c)$. Then $f_{1}(a) \frown_{A} b$ and $101(a) \frown_{A} c$, by (*), which implies that $h(b)=0$ or $h(c)=0$. It now follows that $h \circ\left(\mu \uparrow_{B}\right)^{-1}$ extends to $\mu(\mathbf{A})$.

\section{Dualisable three-element unary algebras that are not strongly dualisable}

In this section, we will show that every algebra that has type $(2)_{Q}$ or type (2) is not strongly dualisable. This will complete our characterisation of the strongly dualisable three-element unary algebras. Our proof is based on the proof by Hyndman and Willard [7] that the unary algebra $\{\{0,1,2\} ; 001,122\rangle$ is not strongly dualisable. Most of the results in this section will also be used in the following section to finish the classification of fully dualisable three-element unary algebras. In our proof, we make use of a special pair of ordered sets.

LEMMA 4.1 ([7, Lemma 4.1]). There is a chain $\Gamma=\langle\Gamma ; \leqslant\rangle$ and an ordered set $\Gamma^{\prime}=\langle\Gamma ; \triangleleft\rangle$ such that $\triangleleft$ is strictly contained in $\leqslant$ and the following condition holds: for all $c, d \in \Gamma$ with $c \leqslant d$ and $c \not d$, there exists $\left\{c_{n} \mid n \in \mathbb{N}\right\} \cup\left\{d_{n} \mid n \in \mathbb{N}\right\} \subseteq \Gamma$ such that $c \triangleleft c_{n}$ and $d_{n} \triangleleft d$ and $c_{n} \leqslant d_{n} \leqslant c_{n+1}$, for every $n \in \mathbb{N}$.

Throughout the rest of this paper, $\boldsymbol{\Gamma}$ and $\boldsymbol{\Gamma}^{\prime}$ will denote a fixed pair of ordered sets satisfying the conditions of Lemma 4.1. The following lemma gives a general method for proving that a finite algebra is not strongly dualisable. Let $\mathscr{G}$ denote the category of directed graphs. 
LEMMA 4.2. Let $\underline{\mathbf{M}}$ be a finite algebra and let $\mathbf{B} \leqslant \mathbf{A}$ in $\mathscr{A}:=\mathbb{N P}(\underline{\mathbf{M}})$ such that $\Gamma \subseteq B$. Assume there is a chain $\mathbf{C}=\langle C ; \leqslant\rangle$, with $C \subseteq M$, for which the maps $-\uparrow_{\Gamma}: \mathscr{A}(\mathbf{A}, \underline{\mathbf{M}}) \rightarrow \mathscr{G}(\mathbf{\Gamma}, \mathbf{C})$ and $-\uparrow_{\Gamma}: \mathscr{A}(\mathbf{B}, \underline{\mathbf{M}}) \rightarrow \mathscr{G}\left(\Gamma^{\prime}, \mathbf{C}\right)$ are well-defined bijections.

(i) The set $X:=\left\{\left.x\right|_{B} \mid x \in \mathscr{A}(\mathbf{A}, \underline{\mathbf{M}})\right\}$ forms a closed substructure of $\underline{\mathbf{M}}^{B}$, for each alter ego $\mathbf{\sim}$ of $\underline{\mathbf{M}}$.

(ii) The algebra $\underline{\mathbf{M}}$ is not strongly dualisable.

ProOF. Let $\mathbf{M}=\langle M ; G, H, R, \mathscr{T}\rangle$ be an alter ego of $\underline{\mathbf{M}}$. There are $c, d \in \Gamma$ with $c \leqslant d$ and $c \nless d$. There also exist $0,1 \in C$ such that $0 \neq 1$ and $0 \leqslant 1$. Define the $\operatorname{map} w: \Gamma \rightarrow C$ by

$$
w(a)= \begin{cases}1 & \text { if } c \triangleleft a ; \\ 0 & \text { otherwise }\end{cases}
$$

Then $w \in \mathscr{G}\left(\boldsymbol{\Gamma}^{\prime}, \mathbf{C}\right)$ and so there is a homomorphism $\bar{w} \in \mathscr{A}(\mathbf{B}, \underline{\mathbf{M}})$ for which $\bar{w} \Gamma_{\Gamma}=w$. As $w \notin \mathscr{G}(\boldsymbol{\Gamma}, \mathbf{C})$, we must have $\bar{w} \notin X$ and therefore $X \neq \mathscr{A}(\mathbf{B}, \underline{\mathbf{M}})$. We will show that $X$ forms a closed substructure of $\mathrm{D}(\mathbf{B}) \leqslant \mathbf{M}^{B}$. As $X$ separates the elements of $B$, it will follow by Theorem 1.2 that $\mathbf{M}$ does not yield a strong duality on $\mathscr{A}$.

Let $\imath: \mathbf{B} \rightarrow \mathbf{A}$ denote the inclusion map. Then $X$ is the image of the morphism $\mathrm{D}(\iota): \mathrm{D}(\mathbf{A}) \rightarrow \mathrm{D}(\mathbf{B})$. This implies that $X$ is topologically closed in $\mathrm{D}(\mathbf{B})$ and that $X$ is closed under the operations in $G$. It remains to check that $X$ is closed under the partial operations in $H$. So let $h \in H$ be a $k$-ary partial operation, for some $k \in \mathbb{N}$, and let $x_{1}, \ldots, x_{k} \in X$ with $\left(x_{1}, \ldots, x_{k}\right) \in \operatorname{dom}(h)^{\mathrm{D}(\mathrm{B})}$. We will show that $z:=h\left(x_{1}, \ldots, x_{k}\right) \in X$.

To show that $z \in X$, it is enough to prove that $\left.z\right|_{\Gamma} \in \mathscr{Y}(\Gamma, \mathrm{C})$. So let $c, d \in \Gamma$ with $c \leqslant d$. Now we wish to show that $z(c) \leqslant z(d)$. Since $z \in \mathscr{A}(\mathbf{B}, \underline{\mathbf{M}})$, we know that $z \Gamma_{\Gamma} \in \mathscr{G}\left(\Gamma^{\prime}, \mathbf{C}\right)$. So we can assume that $c \nless d$. There exists a subset $\left\{c_{n} \mid n \in \mathbb{N}\right\} \cup\left\{d_{n} \mid n \in \mathbb{N}\right\}$ of $\Gamma$ such that $c \triangleleft c_{n}$ and $d_{n} \triangleleft d$ and $c_{n} \leqslant d_{n} \leqslant c_{n+1}$, for all $n \in \mathbb{N}$. We have $\left.x_{i}\right|_{\Gamma} \in \mathscr{G}(\boldsymbol{\Gamma}, \mathbf{C})$, for each $i \in\{1, \ldots, k\}$. As $\mathbf{C}$ is finite, there are $n, m \in \mathbb{N}$, with $n<m$, such that $x_{i}\left(c_{n}\right)=x_{i}\left(c_{m}\right)$, for all $i \in\{1, \ldots, k\}$. Since $c_{n} \leqslant d_{n} \leqslant c_{m}$, we get $x_{i}\left(c_{n}\right)=x_{i}\left(d_{n}\right)$, for all $i \in\{1, \ldots, k\}$, and therefore $z\left(c_{n}\right)=z\left(d_{n}\right)$. As $\left.z\right|_{\Gamma} \in \mathscr{G}\left(\Gamma^{\prime}, \mathbf{C}\right)$, with $c \triangleleft c_{n}$ and $d_{n} \triangleleft d$, it follows that $z(c) \leqslant z\left(c_{n}\right)=z\left(d_{n}\right) \leqslant z(d)$. Thus $z \in X$, whence $\mathbf{X}$ is a closed substructure of $\mathrm{D}(\mathbf{B})$.

Now let $\underline{\mathbf{M}}$ be a unary algebra on the set $\{0,1,2\}$. In order to apply Lemma 4.2 , we shall give a method for constructing algebras in the quasi-variety $\mathscr{A}:=\mathbb{I S P}(\underline{\mathrm{M}})$ using ordered sets. 
DefintTion 4.3. Let $\underline{\mathbf{M}}$ be a unary algebra on the set $\{0,1,2\}$, let $\mathbf{P}=\langle P ; \leqslant\rangle$ be an ordered set and let $\triangleleft$ be a subset of $\leqslant$. Define the set $P^{+}:=P \cup\{T, \perp\}$. For all $a, b \in P$ such that $a \triangleleft b$, define $\overparen{a b} \in M^{P^{+}}$by $\overparen{a b}(\perp)=2, \overparen{a b}(\mathrm{~T})=1$ and

$$
\widehat{a b}(c)= \begin{cases}2 & \text { if } c \leqslant a ; \\ 0 & \text { if } c \leqslant b \text { and } c \nless a \\ 1 & \text { otherwise, }\end{cases}
$$

for all $c \in P$. Define the algebra $\widehat{\mathbf{P}}_{\triangleleft}:=\operatorname{sg}_{\underline{\mathrm{M}}^{p+}}(\{\widehat{a b} \mid a, b \in P$ and $a \triangleleft b\})$. If the relation $\triangleleft$ on $P$ is reflexive, we can define the injection $\iota_{\mathbf{P}}: P \rightarrow \widehat{P}_{\triangleleft}$ by $\iota_{\mathbf{P}}(a):=\overparen{a a}$. If $\triangleleft$ is equal to $\leqslant$, then we write $\widehat{\mathbf{P}}$ instead of $\widehat{\mathbf{P}}_{\triangleleft}$.

The following lemma describes the structure of $\widehat{\mathbf{P}}_{\triangleleft}$ under some special conditions on $\triangleleft$ and $\underline{\mathbf{M}}$. For each set $S$ and each $a \in\{0,1,2\}^{S}$, define the partition

$$
\mathscr{P}(a):=\left\{a^{-1}(0), a^{-1}(1), a^{-1}(2)\right\} \backslash\{\varnothing\}
$$

of $S$. For every algebra $\mathbf{A} \leqslant \underline{\mathbf{M}}^{S}$, the set $A_{\downarrow 2}:=\{a \in A|| \mathscr{P}(a) \mid \leqslant 2\}$ is a subuniverse of $\mathbf{A}$.

LEMMA 4.4. Let $\underline{\mathrm{M}}$ be a unary algebra with type $(2)_{\mathrm{Q}}$. Let $\mathbf{P}=\langle P ; \leqslant\rangle$ be an ordered set, let $\triangleleft$ be a reflexive subset of $\leqslant$ and define $\mathbf{A}:=\widehat{\mathbf{P}}_{\triangleleft}$.

(i) Then $\operatorname{sg}_{\mathrm{A}}(\widehat{a b}) \backslash\{\widehat{a b}, 021(\widehat{a b})\} \subseteq \operatorname{sg}_{\mathrm{A}}(\{\widehat{a a}, \widehat{b b}\})$, for all $a, b \in P$ with $a \triangleleft b$.

(ii) The set of petals of $\mathbf{A}_{\downarrow 2}$ is $\left\{\mathbf{s g}_{\mathbf{A}}(\overparen{a a}) \mid a \in P\right\}$.

PROOF. Let $a, b \in P$ such that $a \triangleleft b$. For all unary term functions $u_{1}$ and $u_{2}$ of $\underline{\mathbf{M}}$, with $\operatorname{ker}\left(u_{1}\right)=\{02 \mid 1\}$ and $\operatorname{ker}\left(u_{2}\right)=\{01 \mid 2\}$, we have $u_{1}(\overparen{a b})=u_{1}(\widehat{b b})$ and $u_{2}(\widehat{a b})=$ $u_{2}(\overparen{a a})$. Using Lemma 3.2, it follows that $\operatorname{sg}_{\mathrm{A}}(\overparen{a b}) \backslash\{\widehat{a b}, 021(\widehat{a b})\} \subseteq \operatorname{sg}_{\mathrm{A}}(\{\widehat{a a}, \widehat{b b}\})$. So claim (i) holds and $A_{\downarrow 2}=\bigcup\left\{\operatorname{sg}_{\mathrm{A}}(\widehat{a a}) \mid a \in P\right\}$.

To prove (ii), it is enough to show that $\operatorname{sg}_{\mathrm{A}}(\widehat{a a}) \cap \operatorname{sg}_{\mathrm{A}}(\overparen{b b})=C_{\mathrm{A}}$, for all $a, b \in P$ with $a \neq b$. Assume that $u(\widehat{a a})=v(\widehat{b b})$, for some $a, b \in P$ with $a \neq b$ and some unary term functions $u$ and $v$ of $\underline{\mathbf{M}}$. Since the two-block partitions $\mathscr{P}(\overparen{a a})$ and $\mathscr{P}(\widehat{b b})$ of $P^{+}$are different, we have $u(\widehat{a a}) \in\{\widehat{0}, \widehat{1}, \widehat{2}\}$. But $\widehat{a a} \in\{1,2\}^{P^{+}}$and $\underline{\mathbf{M}}$ does not have any unary term functions with kernel $\{0 \mid 12\}$. So $u$ is a constant term function of $\underline{\mathrm{M}}$, which implies that $u(\widehat{a a}) \in C_{\mathrm{A}}$.

To illustrate Lemma 4.4, we consider a particular example.

EXAMPLE 4.5. Let $\underline{\mathbf{M}}$ be a unary algebra with type (2) $)_{\mathrm{Q}}$. Define the three-element chain $\mathbf{P}=\langle\{a, b, c\} ; \leqslant\rangle$, such that $a \leqslant b \leqslant c$, and define the ordered set $\mathbf{P}^{\prime}=$ 

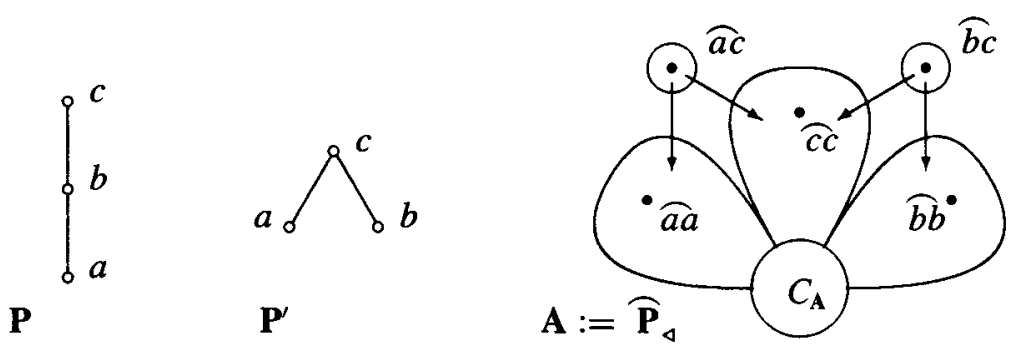

FIGURE 1. Example 4.5

$\langle\{a, b, c\} ; \triangleleft\rangle$, such that $\triangleleft=\leqslant \backslash\{(a, b)\}$. Denote each element $x \in M^{P^{+}}$by the 5-tuple $(x(\perp), x(a), x(b), x(c), x(\mathrm{~T}))$. Then

$$
\begin{gathered}
\widehat{a c}=(2,2,0,0,1), \quad \widehat{b c}=(2,2,2,0,1), \\
\widehat{a a}=(2,2,1,1,1), \quad \widehat{c c}=(2,2,2,2,1), \quad \widehat{b b}=(2,2,2,1,1) .
\end{gathered}
$$

The structure of the algebra $\mathbf{A}:=\widehat{\mathbf{P}}_{\triangleleft}$ is shown in Figure 1. The three petals of $\mathbf{A}_{\downarrow 2}$ $\operatorname{are~} \mathbf{s g}_{\mathbf{A}}(\widehat{a a}), \mathbf{s g}_{\mathbf{A}}(\widehat{b b})$ and $\mathbf{s g}_{\mathbf{A}}(\widehat{c c})$.

The following lemma shows that the algebra $\widehat{\mathbf{P}}_{\triangleleft}$ and the graph $\langle P ; \triangleleft\rangle$ are intimately connected. Define the two-element chain $\underline{2}=\langle\{1,2\} ; \leqslant\rangle$ such that $1 \leqslant 2$. For each set $S$ and $s \in S$, let $\pi_{s}: \underline{\mathbf{M}}^{S} \rightarrow \underline{\mathbf{M}}$ denote the $s$ th projection function.

LEMMA 4.6. Let $\underline{\mathbf{M}}$ be a unary algebra with type $(2)_{\mathrm{Q}}$ and define $\mathscr{A}:=\mathbb{\mathbb { S }} \mathbb{P}(\underline{\mathrm{M}})$. Let $\mathbf{P}=\langle P ; \leqslant\rangle$ be an ordered set, let $\triangleleft$ be a reflexive subset of $\leqslant$ and define $\mathbf{P}^{\prime}=\langle P ; \triangleleft\rangle$.

(i) For all $x \in \mathscr{A}\left(\widehat{\mathbf{P}}_{\triangleleft}, \underline{\mathbf{M}}\right)$ and $a, b \in P$ such that $a \triangleleft b$, we have $x(\widehat{a b})=2$ if and only if $x(\widehat{a a})=2$, and $x(\widehat{a b})=1$ if and only if $x(\widehat{b b})=1$.

(ii) The map - $\iota_{\mathbf{P}}: \mathscr{A}\left(\widehat{\mathbf{P}}_{\triangleleft}, \underline{\mathbf{M}}\right) \rightarrow \mathscr{G}\left(\mathbf{P}^{\prime}, \underline{2}\right)$ is a well-defined bijection.

PROOF. As $\underline{\mathbf{M}}$ has type $(2)_{\mathrm{Q}}$, there are $p, q \in M$, with $p \neq q$, such that both $p p q$ and $q p q$ are term functions of $\underline{\mathbf{M}}$. Define $\mathbf{A}:=\widehat{\mathbf{P}}_{\triangleleft}$. For all $x \in \mathscr{A}(\mathbf{A}, \underline{\mathbf{M}})$ and $a, b \in P$ with $a \triangleleft b$, we have

$$
\begin{array}{rlrlll}
x(\widehat{a b})=2 & \Longleftrightarrow & p p q(x(\widehat{a b}))=q & \Longleftrightarrow & x(p p q(\widehat{a b}))=q \\
& \Longleftrightarrow \quad x(p p q(\widehat{a a}))=q & \Longleftrightarrow & x(\widehat{a a})=2 & &
\end{array}
$$

and, similarly, $x(\widehat{a b})=1$ if and only if $x(\widehat{b b})=1$. So (i) holds. 
We want to define the map $\eta: \mathscr{A}(\mathbf{A}, \underline{\mathbf{M}}) \rightarrow \mathscr{G}\left(\mathbf{P}^{\prime}, \underline{\mathbf{2}}\right)$ by $\eta(x):=x \circ \iota_{\mathbf{P}}$. To see that this will work, let $x \in \mathscr{A}(\mathbf{A}, \underline{\mathbf{M}})$. For each $a \in P$, we have

$$
p p q(x(\widehat{a a}))=x(p p q(\widehat{a a}))=x(q p q(\widehat{a a}))=q p q(x(\widehat{a a})),
$$

which implies that $x(\widehat{a a}) \in\{1,2\}$. Therefore $x \circ \iota_{\mathbf{P}}(P) \subseteq\{1,2\}$. For all $a, b \in P$ such that $a \triangleleft b$ and $x(\widehat{a a})=2$, we have $x(\widehat{a b})=2$ and therefore $x(\widehat{b b})=2$, by (i). Thus $x \circ \iota_{\mathbf{P}} \in \mathscr{G}\left(\mathbf{P}^{\prime}, \underline{2}\right)$ and $\eta$ is well defined.

Let $x, y \in \mathscr{A}(\mathbf{A}, \underline{\mathbf{M}})$ such that $\eta(x)=\eta(y)$ and let $a, b \in P$ with $a \triangleleft b$. Then

$$
x(\widehat{a b})=2 \Longleftrightarrow x(\widehat{a a})=2 \Longleftrightarrow y(\widehat{a a})=2 \Longleftrightarrow y(\widehat{a b})=2,
$$

by (i), and, similarly, $x(\widehat{a b})=1$ if and only if $y(\widehat{a b})=1$. So $x=y$ and $\eta$ is one-to-one.

It remains to show that $\eta$ is onto. Let $z \in \mathscr{G}\left(\mathbf{P}^{\prime}, 2\right)$. By Lemma 4.4 (ii), there is a homomorphism $z_{*}: \mathbf{A}_{12} \rightarrow \underline{\mathbf{M}}$, given by

$$
z_{*}:=\bigsqcup\left\{\left.\pi_{\mathrm{T}}\right|_{\mathrm{sg}_{\mathrm{A}}(a \mathrm{a})} \mid a \in z^{-1}(1)\right\} \cup \bigsqcup\left\{\left.\pi_{\perp}\right|_{\mathrm{sg}_{\mathrm{A}}(a a)} \mid a \in z^{-1}(2)\right\},
$$

such that $z_{*} \circ \iota_{\mathrm{P}}=z$. We will show that $z_{*}$ extends to a homomorphism $\bar{z}: \mathbf{A} \rightarrow$ M. Let $a, b \in P$ such that $a \neq b$ and $a \triangleleft b$. We want to find $c \in P^{+}$with

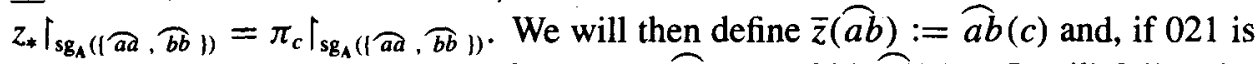
a term function of $\underline{\mathrm{M}}$, we will define $\bar{z}(021(\widehat{a b})):=021(\widehat{a b})(c)$. It will follow by Lemma 4.4 (i) that $z_{*}$ extends to $A$.

We can assume that $z(a) \neq z(b)$. Therefore $z(a)=1$ and $z(b)=2$, as $z(a) \leqslant z(b)$. We have $z_{*}(\widehat{a a})=\pi_{\uparrow}(\widehat{a a})=1=\widehat{a a}(b)$ and $z_{*}(\widehat{b b})=\pi_{\perp}(\widehat{b b})=2=\widehat{b b}(b)$, which implies that $\left.z_{*}\right|_{\left.\mathbf{s g}_{A}((a), \widehat{b b})\right)}=\left.\pi_{b}\right|_{\mathbf{s g}_{A}(\mid \overparen{a d}, \widehat{b b})}$. Thus $\eta$ is a bijection.

THEOREM 4.7. Let $\underline{\mathbf{M}}$ be a unary algebra with type $(2)_{\mathrm{Q}}$. Then $\underline{\mathbf{M}}$ is not strongly dualisable.

PROOF. Define $\mathscr{A}:=$ DSP(M). Using Lemma 4.1 and Definition 4.3, we have algebras $\widehat{\Gamma}_{\triangleleft} \leqslant \overparen{\Gamma}$ in $\mathscr{A}$ and an injection $\iota_{\Gamma}: \Gamma \rightarrow \widehat{\Gamma}_{\triangleleft}$. By Lemma 4.6 (ii), the maps $-\circ \iota_{\Gamma}: \mathscr{A}(\widehat{\Gamma}, \underline{\mathbf{M}}) \rightarrow \mathscr{G}(\boldsymbol{\Gamma}, \underline{2})$ and $-\circ \iota_{\mathbf{\Gamma}}: \mathscr{A}\left(\widehat{\boldsymbol{\Gamma}}_{\triangleleft}, \underline{\mathbf{M}}\right) \rightarrow \mathscr{G}\left(\boldsymbol{\Gamma}^{\prime}, \underline{2}\right)$ are well-defined bijections. It follows by Lemma 4.2 (ii) that $\underline{\mathrm{M}}$ is not strongly dualisable.

The algebra $\underline{\mathbf{M}}^{\sharp}=\left\langle\{0,1,2\} ; F^{\sharp}\right\rangle$, defined near the beginning of Section 3 , has type (2) $)_{Q}$. Every two-kernel algebra, with kernels $\{01 \mid 2\}$ and $\{02 \mid 1\}$, is a reduct of $\underline{\mathbf{M}}^{\sharp}$. We will use $\underline{\mathbf{M}}^{\sharp}$ to prove that none of the algebras of type $(2)_{C}$ are strongly dualisable.

LEMMA 4.8. Let $\mathrm{M}$ be a unary algebra with type (2) $\mathrm{C}$. Let $\mathbf{A}$ be an algebra in $\mathscr{A}^{\sharp}:=\mathbb{B S P}\left(\underline{\mathbf{M}}^{\sharp}\right)$ and let $\mathbf{A}^{\mathrm{b}}$ be the reduct of $\mathbf{A}$ in $\mathscr{A}:=\mathbb{N S P}(\underline{\mathbf{M}})$. Then $\mathscr{A}\left(\mathbf{A}^{\mathrm{b}}, \underline{\mathbf{M}}\right)=$ $\mathscr{A}^{\sharp}\left(\mathbf{A}, \underline{\mathbf{M}}^{\sharp}\right)$. 
PROOF. We begin by proving that the only homomorphisms from $\underline{M}^{2}$ to $\underline{M}$ are the two projections. Let $x: \underline{\mathbf{M}}^{2} \rightarrow \underline{\mathbf{M}}$ be a homomorphism. Then $x(0,1) \in\{0,1\}$, since $x$ preserves $010=101 \circ 101$. First assume that $x(0,1)=1$. In $\underline{\mathbf{M}}^{2}$, we have
$(0,1)$
$\stackrel{101}{\longleftrightarrow}$
$(1,0)$
$\stackrel{101}{\longleftarrow}$
$(2,1) \stackrel{220}{\longrightarrow}$
$(0,2)$
$\stackrel{220}{\longleftrightarrow}$
$(2,0) \longleftarrow 220$
$(1,2)$.

Applying the homomorphism $x$ gives us

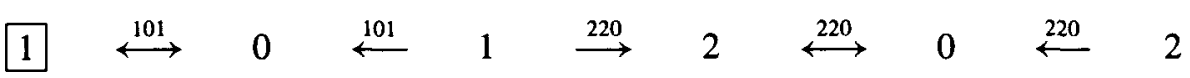

in $\underline{\mathbf{M}}$. The constant operations 000,111 and 222 are all term functions of $\underline{\mathbf{M}}$. So it follows that $x=\pi_{2}$. Now assume that $x(0,1)=0$. Then $x(1,0)=101(x(0,1))=1$ and, by symmetry, we have $x=\pi_{1}$.

We can assume that $\mathbf{A} \leqslant\left(\underline{\mathbf{M}}^{\sharp}\right)^{S}$, for some non-empty set $S$. Let $a \in A$ such that $\mathscr{P}(a)$ has two blocks. Then $\operatorname{sg}_{\mathbf{A}}(a)=\left\{b \in M^{S} \mid \mathscr{P}(b)=\mathscr{P}(a)\right.$ or $\left.\mathscr{P}(b)=\{S\}\right\}$, as every map in $F^{\sharp}$ is an operation of $\underline{\mathbf{M}}^{\sharp}$. Let $\mathbf{s g}_{\mathrm{A}}(a)^{b}$ denote the reduct of $\mathbf{s g}_{\mathrm{A}}(a)$ in $\mathscr{A}$. It now follows that $\operatorname{sg}_{\mathrm{A}}(a)^{\mathrm{b}}$ is isomorphic to $\underline{\mathbf{M}}^{2}$, via repetition of coordinates. Since the only homomorphisms from $\underline{\mathbf{M}}^{2}$ to $\underline{\mathbf{M}}$ are the projections, every homomorphism from $\operatorname{sg}_{\mathrm{A}}(a)^{\mathrm{b}}$ to $\underline{\mathrm{M}}$ is the restriction of a projection.

Now let $y \in \mathscr{A}\left(\mathbf{A}^{\mathrm{b}}, \underline{\mathbf{M}}\right)$ and let $a \in A$. To prove that $y \in \mathscr{A}^{\sharp}\left(\mathbf{A}, \underline{\mathbf{M}}^{\sharp}\right)$, it suffices to show that $y \Gamma_{\mathrm{sg}_{\mathrm{A}}(a)}$ is the restriction of a projection. The constant maps 000,111 and 222 are all term functions of $\mathbf{M}$. So, if $a \in\{\widehat{0}, \widehat{1}, \widehat{2}\}$, then $\left.y\right|_{\mathrm{sg}_{\Lambda}(a)}$ is the restriction of a projection. If $a \in A_{\downarrow 2} \backslash\{\widehat{0}, \widehat{1}, \widehat{2}\}$, then $\mathscr{P}(a)$ has two blocks and so we know that $\left.y\right|_{\mathrm{sg}_{\Lambda}(a)}$ is the restriction of a projection. Therefore we can assume that $a \in A \backslash A_{\downarrow 2}$. For each $m \in\{1,2\}$, define $P_{m}:=\operatorname{sg}_{\mathrm{A}}\left(f_{m}(a)\right)$. Then $\operatorname{sg}_{\mathrm{A}}(a)=P_{1} \cup P_{2} \cup\{a, 021(a)\}$. Since $|\mathscr{P}(a)|=3$, we can choose some $s \in a^{-1}(y(a))$. For each $m \in\{1,2\}$, we have $y\left(f_{m}(a)\right)=f_{m}(y(a))=f_{m}(a(s))$ and so $y \uparrow_{P_{m}}=\pi_{s} \uparrow_{P_{m}}$, as $y \uparrow_{P_{m}}$ is the restriction of a projection and all the elements of $P_{m} \backslash\{\widehat{0}, \widehat{1}, \widehat{2}\}$ determine the same partition of $S$. For each $m \in\{1,2\}$, we have $f_{m} \circ 021(a) \in P_{1} \cup P_{2}$ and so $f_{m}(y(021(a)))=$ $y\left(f_{m}(021(a))\right)=f_{m}(021(a(s)))$. Therefore $y(021(a))=021(a(s))$, since $f_{1}$ and $f_{2}$ separate $M$. We have shown that $\left.y\right|_{\mathbf{s g}_{\mathcal{A}_{A}}(a)}=\left.\pi_{s}\right|_{\mathbf{s g}_{\mathbf{A}}(a)}$, whence $y \in \mathscr{A}^{\sharp}\left(\mathbf{A}, \underline{\mathbf{M}}^{\sharp}\right)$.

THEOREM 4.9. Let $\underline{\mathbf{M}}$ be a unary algebra with type (2). Then $\underline{\mathbf{M}}$ is not strongly dualisable.

ProOF. Define $\mathscr{A}:=\mathbb{N S P}(\underline{\mathbf{M}})$ and $\mathscr{A} \sharp:=\mathbb{S} \mathbb{P}\left(\underline{\mathbf{M}}^{\sharp}\right)$. Using Lemma 4.1 and Definition 4.3, we have $\widehat{\Gamma}_{\triangleleft} \leqslant \widehat{\Gamma}$ in $\mathscr{A}^{\sharp}$ and an injection $\iota_{\Gamma}: \Gamma \rightarrow \widehat{\Gamma}_{\triangleleft}$. The maps $-\circ \iota_{\Gamma}: \mathscr{A}^{\sharp}\left(\widehat{\Gamma}, \underline{\mathbf{M}}^{\sharp}\right) \rightarrow \mathscr{G}(\boldsymbol{\Gamma}, \underline{2})$ and $-\circ \iota_{\Gamma}: \mathscr{A}^{\sharp}\left(\widehat{\boldsymbol{\Gamma}}_{\triangleleft}, \underline{\mathbf{M}}^{\sharp}\right) \rightarrow \mathscr{G}\left(\boldsymbol{\Gamma}^{\prime}, \underline{2}\right)$ are welldefined bijections, by Lemma 4.6 (ii). Let $\widehat{\Gamma}^{b}$ and $\widehat{\Gamma}_{\triangleleft}^{b}$ denote the reducts of $\widehat{\Gamma}$ and $\widehat{\Gamma}_{\triangleleft}$ in the quasi-variety $\mathscr{A}$. Then, by Lemma 4.8 , the maps $-\circ \iota_{\Gamma}: \mathscr{A}\left(\widehat{\Gamma}^{\mathrm{b}}, \underline{\mathrm{M}}\right) \rightarrow$ $\mathscr{G}(\boldsymbol{\Gamma}, \underline{2})$ and $-\circ \iota_{\Gamma}: \mathscr{A}\left(\widehat{\mathbf{\Gamma}}_{\triangleleft}^{\mathrm{b}}, \underline{\mathbf{M}}\right) \rightarrow \mathscr{G}\left(\boldsymbol{\Gamma}^{\prime}, \underline{2}\right)$ are bijections. So Lemma 4.2 (ii) tells us that $\underline{\mathbf{M}}$ is not strongly dualisable. 
We have now finished establishing the classification of strongly dualisable threeelement unary algebras given in the introduction. Part (i) of the characterisation follows from Theorem 2.6. It is shown in [4] that each unary algebra with type (2) $P$ is not dualisable. So part (ii) of the characterisation follows from Theorems 3.4, 3.8, 3.10, 4.7 and 4.9. The three-kernel three-element unary algebras are shown to be not dualisable, and therefore not strongly dualisable, in [4].

LEMMA 4.10. Let $\underline{\mathrm{M}}$ be a unary algebra with type $(2)_{\mathrm{Q}}$ or type $(2)_{\mathrm{C}}$. Then $\underline{\mathrm{M}}$ is not $n$-quasi-injective, for all $n \in \mathbb{N}$.

ProOF. First assume that $\underline{\mathbf{M}}$ has type (2) Q $_{\text {. Let }} n \in \mathbb{N}$ and define $k:=2 n+1$. Let $\mathbf{D}=\langle\{0, \ldots, k\} ; \leqslant\rangle$ be a $(k+1)$-element chain with $0 \leqslant \cdots \leqslant k$. Define the relations $\varangle:=\{(i, i) \mid i \in D\} \cup\{(i, i+1) \mid i \in D \backslash\{k\}\}$ and $\triangleleft:=\varangle \backslash\{(n, n+1)\}$ on $D$. Using Definition 4.3 , we can define $\mathbf{A}:=\widehat{\mathbf{D}} \triangleleft$ and $\mathbf{C}:=\widehat{\mathbf{D}}_{\triangleleft}$, where $\mathbf{C}$ is a subalgebra of A. By Lemma 4.4 (ii), the coproduct $\mathbf{B}:=\mathbf{s g}_{\mathbf{A}}(\widehat{00}) * \mathbf{s g}_{\mathbf{A}}(\widehat{k k})$ is also a subalgebra of A. Define the homomorphism $x: \mathbf{B} \rightarrow \underline{\mathbf{M}}$ by $x:=\pi_{\perp} l_{\mathrm{sg}_{A}(\widehat{00})} \sqcup \pi_{T} l_{\mathrm{sg}_{\mathrm{A}}(\widehat{k k})}$. Then $x \circ \iota_{\mathbf{D}}(0)=x(\widehat{00})=2$ and $x \circ \iota_{\mathbf{D}}(k)=x(\widehat{k k})=1$. To prove that $\underline{\mathbf{M}}$ is not $n$-quasi-injective, we shall show that $x$ extends to $n_{\mathrm{A}}(B)$ but not to $A$.

The map $x \circ \iota_{D}\left\lceil_{\{0, k\}}:\{0, k\} \rightarrow\{1,2\}\right.$ does not extend to a morphism from $\langle D ; 4\rangle$ to $\underline{2}$. So, by Lemma 4.6 (ii), the homomorphism $x: B \rightarrow \underline{M}$ does not extend to $A$. There is an extension $y:\langle D ; \triangleleft\rangle \rightarrow \underline{\mathbf{2}}$ of $\left.x \circ \iota_{\mathbf{D}}\right|_{\{0, k\}}$. Using Lemma 4.6 (ii) again, there is a homomorphism $\bar{y}: \mathbf{C} \rightarrow \underline{\mathbf{M}}$ such that $\bar{y} \circ \iota_{\mathbf{D}}=y$. We have $\bar{y}(\widehat{00})=x(\widehat{00})$ and $\bar{y}(\overparen{k k})=x(\overparen{k k})$. So $\bar{y}$ is an extension of $x$. We have shown that $x$ extends to $\mathbf{C}$ but not to $\mathbf{A}$. It remains to prove that $n_{\mathrm{A}}(B) \subseteq C$.

Define $n^{\prime}:=n+1$. Then $A \backslash C \subseteq\left\{\widehat{n n}^{\prime}, 021\left(\widehat{n} n^{\prime}\right)\right\}$, by Lemma 4.4 (i). Using Figure 2 and Lemma 4.4, the reader can check that $d_{A}(a, b) \geqslant n+1$, for all $a \in A \backslash C$ and $b \in B \backslash C_{\mathrm{A}}$. Thus $n_{\mathrm{A}}(B) \subseteq C$, whence $\underline{\mathrm{M}}$ is not $n$-quasi-injective.

Now assume that $\underline{\mathrm{M}}$ has type $(2)_{\mathrm{C}}$ and let $n \in \mathbb{N}$. We have just shown that $\underline{\mathrm{M}}^{\sharp}$ is

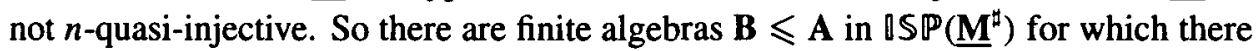
is a homomorphism $x: \mathbf{B} \rightarrow \underline{\mathbf{M}}^{\sharp}$ that extends to $n_{\mathbf{A}}(B)$ but not to $\mathbf{A}$. Let $\mathbf{A}^{b}$ denote

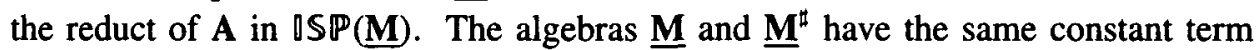
functions. So $C_{\mathrm{A}^{p}}=C_{\mathrm{A}}$. It follows that $n_{\mathrm{A}^{p}}(B) \subseteq n_{\mathrm{A}}(\dot{B})$. Thus $x$ extends to $n_{\mathrm{A}^{p}}(B)$ but not to $\mathbf{A}^{\mathrm{b}}$, using Lemma 4.8 .

The Quasi-injectivity Theorem, given in the introduction, now follows from Lemma 2.7, Theorem 3.4, Lemma 3.7, Lemma 3.9 and Lemma 4.10.

\section{Dualisable three-element unary algebras that are not fully dualisable}

In this section, we prove that each unary algebra with type $(2)_{Q}$ or type $(2)_{C}$ is not fully dualisable. It will then follow that every fully dualisable three-element unary 


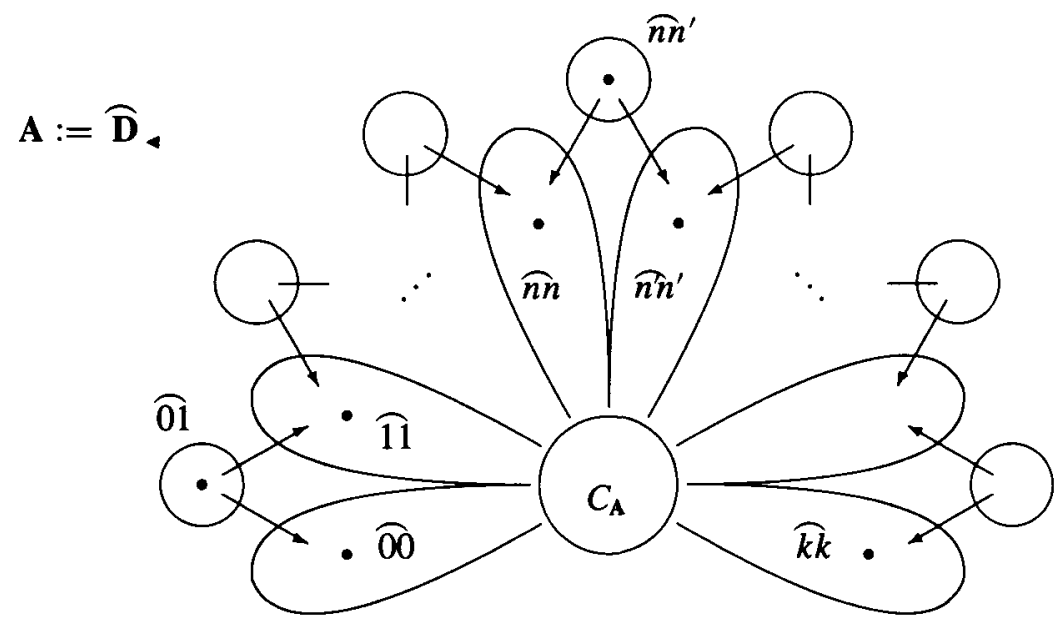

FIGURE 2. Lemma 4.10

algebra is strongly dualisable. Our proof is an extension of that given by Hyndman and Willard [7] to show that the unary algebra $\langle\{0,1,2\} ; 001,122)$ is not fully dualisable. The proof in [7] used the fact that both the operations 001 and 122 preserve the total order with $0 \leqslant 1 \leqslant 2$. Our proof is more complicated since it must work, in particular, for the algebra $\underline{\mathbf{M}}^{\sharp}$, and there is no total order on $\{0,1,2\}$ that is preserved by every operation in $F^{\sharp}$.

A full duality for a quasi-variety $\mathscr{A}:=\mathbb{S} \mathbb{P}(\underline{\mathbf{M}})$ is more subtle than either a duality or a strong duality. At the moment, we have no reason to believe that, if $\mathbf{M}$ is a structure that yields a full duality on $\mathscr{A}$, then every extension of $\mathbf{M}$, via algebraic relations, also yields a full duality on $\mathscr{A}$. However, there are some relations that can always be added to a structure $\underset{\sim}{\mathbf{M}}$ without destroying a full duality. The following lemma is proved in [7], by Hyndman and Willard.

Let $n \in \mathbb{N}$ and consider an $n$-ary algebraic relation $r$ on $\underline{\mathbf{M}}$. Then $r$ is the underlying set of a subalgebra $\mathbf{r}$ of $\underline{\mathbf{M}}^{n}$. The relation $r$ is said to be balanced if $\mathscr{A}(\mathbf{r}, \underline{\mathbf{M}})=\left\{\left.\pi_{i}\right|_{r} \mid i \in\{1, \ldots, n\}\right\}$, and $\left.\pi_{i}\right|_{r} \neq\left.\pi_{j}\right|_{r}$, for all $i, j \in\{1, \ldots, n\}$ with $i \neq j$.

LEMMA 5.1. [7, Lemma 4.7] Let $\mathbf{M}$ be a finite algebra and assume that the structure $\underset{\sim}{\mathbf{M}}=\langle M ; G, H, R, \mathscr{T}\rangle$ yields a full duality on $\mathrm{DS} \mathbb{P}(\underline{\mathrm{M}})$. Let $r$ be a balanced algebraic relation on $\underline{\mathbf{M}}$. Then $\underline{\mathbf{M}}^{\prime}:=\langle M ; G, H, R \cup\{r\}, \mathscr{T}\rangle$ also yields a full duality on $\operatorname{DSP}(\mathbf{M})$.

Now assume that $\underline{M}$ is a unary algebra of type $(2)_{Q}$ or type $(2)_{C}$. Let $\preccurlyeq$ denote the order on $\{0,1,2\}$ with $1 \preccurlyeq 0 \preccurlyeq 2$. We will define some algebraic relations on $\underline{\mathbf{M}}$. The 
definitions of these relations will depend on the type of $\underline{\mathbf{M}}$. If $\underline{\mathbf{M}}$ has type $(2)_{\mathrm{Q}}$, then we define the algebraic relations on $\underline{\mathbf{M}}$ by

$$
\preccurlyeq_{n}:=\operatorname{sg}_{\underline{\underline{M}}^{n}}\left(\left\{a \in M^{n} \mid 1=a(1) \preccurlyeq \cdots \preccurlyeq a(n)=2\right\}\right),
$$

for all $n \in \mathbb{N} \backslash\{1\}$, and

$$
\bowtie:=\operatorname{sg}_{\underline{M}^{6}}\left(\left\{a \in M^{6} \mid 1=a(1) \preccurlyeq \cdots \preccurlyeq a(6)=2\right\rfloor \backslash\{(1,1,0,0,2,2)\}\right) .
$$

If $\underline{\mathbf{M}}$ has type (2) $)_{\mathrm{C}}$ but not type $(2)_{\mathrm{Q}}$, then, since $\underline{\mathbf{M}}$ is a reduct of $\underline{\mathbf{M}}^{\sharp}$, we can define the algebraic relations on $\underline{\mathbf{M}}$ by

$$
\preccurlyeq_{n}:=\operatorname{sg}_{\left(\underline{M}^{n}\right)^{n}}\left(\left\{a \in M^{n} \mid 1=a(1) \preccurlyeq \cdots \preccurlyeq a(n)=2\right\}\right),
$$

for all $n \in \mathbb{N} \backslash\{1\}$, and

$$
\bowtie:=\operatorname{sg}_{\left(\underline{M}^{n}\right)^{6}}\left(\left\{a \in M^{6} \mid 1=a(1) \preccurlyeq \cdots \preccurlyeq a(6)=2\right\} \backslash\{(1,1,0,0,2,2)\}\right) .
$$

The relations $\preccurlyeq_{4}$ and $\bowtie$ will play an important role in our proof that $\underline{\mathbf{M}}$ is not fully dualisable.

LEMMA 5.2. Let $\underline{\mathrm{M}}$ be a unary algebra with type $(2)_{\mathrm{Q}}$ or type $(2)_{\mathrm{C}}$. The relations $\preccurlyeq_{4}$ and $\bowtie$ on $\underline{\mathrm{M}}$ are balanced.

ProOF. First assume that $\underline{\mathbf{M}}$ has type $(2)_{\mathrm{Q}}$ and define $\mathscr{A}:=\mathbb{V S P}(\underline{\mathbf{M}})$. Define the three-element chain $\mathbf{C}=\langle\{0,1,2\} ; \leqslant\rangle$ such that $0 \leqslant 1 \leqslant 2$. Using Definition 4.3 , we have

$$
\widehat{\mathrm{C}}=\mathbf{s g}_{\underline{\mathrm{M}}^{\mathrm{C}^{+}}}\left(\left\{a \in M^{C^{+}} \mid 1=a(\mathrm{~T}) \preccurlyeq a(2) \preccurlyeq a(1) \preccurlyeq a(0)=a(\perp)=2\right\}\right) .
$$

So $\widehat{\mathrm{C}}$ is isomorphic to the algebra $\preccurlyeq_{4}$. By Lemma 4.6 (ii), we have $\left|\mathscr{A}\left(\preccurlyeq_{4}, \underline{\mathrm{M}}\right)\right|=$ $|\mathscr{A}(\widehat{\mathrm{C}}, \underline{\mathrm{M}})|=|\mathscr{G}(\mathbf{C}, \underline{2})|=4$ and therefore $\preccurlyeq_{4}$ is balanced.

Define the five-element chain $\mathbf{D}=\langle\{0,1,2,3,4\} ; \leqslant\rangle$ with $0 \leqslant \cdots \leqslant 4$. Define the graph $\mathbf{D}^{\prime}=\langle D ; \triangleleft\rangle$, where $\triangleleft:=\leqslant \backslash\{(1,3)\}$. Write each element $a \in M^{D^{+}}$as the 7-tuple $(a(\mathrm{~T}), a(4), \ldots, a(0), a(\perp))$. Then

$$
\begin{array}{r}
\widehat{\mathbf{D}}_{\triangleleft}=\mathbf{s g}_{\underline{\mathrm{M}}^{p^{+}}}\left(\left\{a \in M^{D^{+}} \mid 1=a(\mathrm{~T}) \preccurlyeq a(4) \preccurlyeq \cdots \preccurlyeq a(0)=a(\perp)=2\right\} \backslash\right. \\
\\
\{(1,1,0,0,2,2,2)\}),
\end{array}
$$

and $\widehat{\mathbf{D}}_{\triangleleft}$ is isomorphic to the algebra $\triangleleft$. Using Lemma 4.6 (ii), this implies that $|\mathscr{A}(\bowtie, \underline{\mathbf{M}})|=\left|\mathscr{A}\left(\widehat{\mathbf{D}}_{\triangleleft}, \underline{\mathbf{M}}\right)\right|=\left|\mathscr{G}\left(\mathbf{D}^{\prime}, \underline{2}\right)\right|=6$. Thus $\bowtie$ is balanced.

Now assume that $\underline{\mathrm{M}}$ has type $(2)_{\mathrm{C}}$ but not type $(2)_{\mathrm{Q}}$. The relations $\preccurlyeq_{4}$ and $\bowtie$, defined on $\underline{\mathbf{M}}$, are algebraic on $\underline{\mathbf{M}}^{\sharp}$. We have just shown that $\preccurlyeq_{4}$ and $\bowtie$ are balanced on $\underline{\mathbf{M}}^{\sharp}$. So $\preccurlyeq_{4}$ and $\bowtie$ are balanced on $\underline{\mathbf{M}}$, by Lemma 4.8 . 
We will work with the relations $\preccurlyeq_{n}$, for each $n \in \mathbb{N} \backslash\{1\}$, and $\bowtie$ using the properties given in the following lemma.

LEMMA 5.3. Let $\underline{\mathrm{M}}$ be a unary algebra of type $(2)_{\mathrm{Q}}$ or type (2) $\mathrm{C}$. Let $m, n \in \mathbb{N} \backslash\{1\}$ and let $a_{1}, \ldots, a_{n} \in\{0,1,2\}$.

(i) Let $i, j, k \in\{1, \ldots, n\}$ such that $i \leqslant j \leqslant k$. If $a_{i}=a_{k}$ and $a_{i} \neq a_{j}$, then $\left(a_{1}, \ldots, a_{n}\right) \notin \preccurlyeq_{n}$ and $\left(a_{1}, \ldots, a_{n}\right) \notin \bowtie$.

(ii) Let $\sigma:\{1, \ldots, m\} \rightarrow\{1, \ldots, n\}$ such that $\sigma(1)=1, \sigma(m)=n$ and $\sigma$ preserves the natural order. If $\left(a_{1}, \ldots, a_{n}\right) \in \preccurlyeq_{n}$, then $\left(a_{\sigma(1)}, \ldots, a_{\sigma(m)}\right) \in \preccurlyeq_{m}$.

(iii) We have $\preccurlyeq_{6} \backslash \bowtie \subseteq\{(1,1,0,0,2,2),(2,2,0,0,1,1)\} \subseteq M^{6} \backslash \bowtie$.

PROOF. The three claims follow from the definitions, since all the unary term functions of $\underline{\mathrm{M}}$ belong to the set $F^{\sharp}$.

Assume that $\underline{\mathbf{M}}$ has type $(2)_{\mathrm{Q}}$. The algebras $\overparen{\Gamma}$ and $\widehat{\Gamma}_{\triangleleft}$ in $\mathscr{A}:=\operatorname{DSP}(\underline{\mathrm{M}})$ are given by Lemma 4.1 and Definition 4.3 in the previous section. Let $\underset{\sim}{\mathbf{M}}$ be an alter ego of $\underline{M}$ that has $\preccurlyeq_{4}$ and $\bowtie$ in its type. By Lemma 4.2 (i) and Lemma 4.6 (ii), we know that $X:=\{x|\widehat{T} \triangleleft| x \in \mathscr{A}(\widehat{\boldsymbol{\Gamma}}, \underline{\mathbf{M}})\}$ determines a closed substructure $\mathbf{X}$ of $\mathbb{\sim}^{\Gamma} \triangleleft$. To show that $\mathbf{M}$ does not yield a full duality on $\mathscr{A}$, we shall prove, via a sequence of technical lemmas, that $\mathbf{X}$ is not isomorphic to the dual of any algebra in $\mathscr{A}$.

Now assume that $\underline{\mathbf{M}}$ has type $(2)_{\mathrm{Q}}$ or type $(2)_{\mathrm{C}}$. We will associate a graph with each algebra in $\operatorname{USP}(\underline{\mathbf{M}})$. Let $\mathbf{A} \leqslant \underline{\mathbf{M}}^{S}$, for some non-empty set $S$. Recall that $A_{\downarrow 2}:=\{a \in A|| \mathscr{P}(a) \mid \leqslant 2\}$ determines a subalgebra of $\mathbf{A}$, where we define the partition $\mathscr{P}(a):=\left\{a^{-1}(0), a^{-1}(1), a^{-1}(2)\right\} \backslash\{\varnothing\}$ of $S$, for each $a \in A$. For each two-block partition $\mathscr{Q}$ of $S$, we define the subuniverse of $\mathbf{A}_{\downarrow 2}$ determined by $\mathscr{Q}$ to be

$$
A_{\mathscr{Q}}:=\{a \in A \mid \mathscr{P}(a)=\mathscr{Q} \text { or } \mathscr{P}(a)=\{S\}\} .
$$

Define the set

$$
\mathscr{P}_{\mathrm{A}}:=\left\{A_{\mathscr{Q}} \mid \mathscr{Q}=\mathscr{P}(a) \text { for some } a \in A_{\downarrow 2} \backslash\{\hat{0}, \widehat{1}, \widehat{2}\}\right\} .
$$

Then $\mathscr{P}_{\mathbf{A}}$ contains all the partition-determined subuniverses of $\mathbf{A}_{\downarrow 2}$ that do not lie in the set $\{\widehat{0}, \widehat{1}, \widehat{2}\}$. There are unary term functions $u_{1}$ and $u_{2}$ of $\underline{\mathbf{M}}$ such that $\operatorname{ker}\left(u_{1}\right)=\{02 \mid 1\}$ and $\operatorname{ker}\left(u_{2}\right)=\{01 \mid 2\}$. Let $t \in S$ and define the reflexive binary relation $\vec{\rightarrow}_{\mathrm{A}}$ on $\mathscr{P}_{\mathrm{A}}$ such that $P \rightarrow_{\mathrm{A}} Q$ if and only if $P=Q$ or there exists $a \in A \backslash A_{\downarrow 2}$ and $\{m, \widetilde{m}\}=\{1,2\}$ with $u_{m}(a) \in P, u_{\tilde{m}}(a) \in Q$ and $a(t)=m$. The definition of $\rightarrow_{\mathrm{A}}$ is independent of our choice of $u_{1}$ and $u_{2}$. Let $\vec{l}_{\mathrm{A}}$ denote the transitive closure of $\vec{\rightarrow}_{\mathbf{A}}$. Then $\vec{t}_{\mathrm{A}}$ is a quasi-order on $\mathscr{P}_{\mathbf{A}}$. To illustrate the definition of $\vec{\rightarrow}_{\mathbf{A}}$, we revisit the algebra constructed in Example 4.5.

EXAMPLE 5.4. Let $\underline{\mathbf{M}}$ be a unary algebra with type $(2)_{\mathrm{Q}}$. Define the ordered set $\mathbf{P}^{\prime}$ and the algebra $\mathbf{A}:=\widehat{\mathbf{P}}_{\triangleleft}$ as in Example 4.5. We shall show that $\left.\left\langle\mathscr{P}_{\mathbf{A}} ; \longrightarrow_{\mathbf{I}}\right\rangle\right)$ 
is isomorphic to $\mathbf{P}^{\prime}$. Let $u_{1}$ and $u_{2}$ be unary term functions of $\underline{\mathbf{M}}$ with $\operatorname{ker}\left(u_{1}\right)=$ $\{02 \mid 1\}$ and $\operatorname{ker}\left(u_{2}\right)=\{01 \mid 2\}$. The partitions $\mathscr{P}(\widehat{a a}), \mathscr{P}(\widehat{b b})$ and $\mathscr{P}(\widehat{c c})$ of $P^{+}$are distinct. So, using Lemma 4.4 (ii), we have $\mathscr{P}_{\mathrm{A}}=\left\{\operatorname{sg}_{\mathrm{A}}(\widehat{a a}), \mathrm{sg}_{\mathrm{A}}(\overparen{b b}), \mathrm{sg}_{\mathrm{A}}(\widehat{c c})\right\}$. By Lemma 4.4 (i), we get

$$
A \backslash A_{\downarrow 2} \subseteq\{\widehat{a c}, \widehat{b c}, 021(\widehat{a c}), 021(\widehat{b c})\} .
$$

Now $\widehat{a c}(\perp)=2$, with $u_{2}(\widehat{a c})=u_{2}(\widehat{a a}) \in \operatorname{sg}_{\mathrm{A}}(\widehat{a a})$ and $u_{1}(\widehat{a c})=u_{1}(\widehat{c c}) \epsilon$ $\operatorname{sg}_{\mathbf{A}}(\widehat{c c})$, which implies that $\operatorname{sg}_{\mathbf{A}}(\widehat{a a}) \longrightarrow_{\perp} \operatorname{sg}_{\mathbf{A}}(\widehat{c c})$. If 021 is a term function of $\underline{\mathbf{M}}$, then we have $021(\widehat{a c})(\perp)=1$, with $u_{1}(021(\widehat{a c})) \in \operatorname{sg}_{\mathrm{A}}(\widehat{a a})$ and $u_{2}(021(\widehat{a c})) \in$ $\operatorname{sg}_{\mathrm{A}}(\widehat{c c})$, which also implies that $\operatorname{sg}_{\mathrm{A}}(\overparen{a a}) \longrightarrow_{\mathrm{A}} \mathrm{sg}_{\mathrm{A}}(\widehat{c c})$. Similarly, using $\widehat{b c}$ and $021(\widehat{b c})$, we have $\operatorname{sg}_{\mathbf{A}}(\widehat{b b}) \longrightarrow_{\perp} \operatorname{sg}_{\mathbf{A}}(\widehat{c c})$. So there is an isomorphism $\vartheta:\langle P ; \triangleleft\rangle \rightarrow$ $\left\langle\mathscr{P}_{\mathrm{A}} ; \longrightarrow_{\perp}\right\rangle$ given by $\vartheta(x)=\operatorname{sg}_{\mathrm{A}}(\widehat{x x})$.

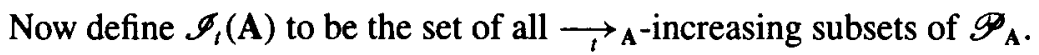

LEMMA 5.5. Let $\underline{\mathrm{M}}$ be a unary algebra of type (2) ${ }_{\mathrm{Q}}$ or type (2) $\mathrm{C}$, and define the quasi-variety $\mathscr{A}:=\mathbb{D S P}(\underline{\mathbf{M}})$. Let $\mathbf{A} \leqslant \underline{\mathbf{M}}^{s}$, with $\mathscr{P}_{\mathbf{A}} \neq \varnothing$, and let $s, t \in S$. Assume that $\left.x\right|_{P}=\left.\pi_{s}\right|_{P}$ or $\left.x\right|_{P}=\left.\pi_{t}\right|_{P}$, for all $x \in \mathscr{A}(\mathbf{A}, \underline{\mathbf{M}})$ and all $P \in \mathscr{P}_{\mathbf{A}}$. Then the map $\eta: \mathscr{A}(\mathbf{A}, \underline{\mathbf{M}}) \rightarrow \mathscr{I}_{1}(\mathbf{A})$, given by $\eta(x):=\left\{P \in \mathscr{P}_{\mathbf{A}}|x|_{P}=\left.\pi_{i}\right|_{P}\right\}$, is a well-defined bijection.

PROOF. There are unary term functions $u_{1}$ and $u_{2}$ of $\underline{\mathbf{M}}$ with $\operatorname{ker}\left(u_{1}\right)=\{02 \mid 1\}$ and $\operatorname{ker}\left(u_{2}\right)=\{01 \mid 2\}$. To see that $\eta$ is well defined, let $x \in \mathscr{A}(\mathbf{A}, \underline{\mathbf{M}})$ and choose $P, Q \in \mathscr{P}_{\mathrm{A}}$, with $P \neq Q$, such that $P \in \eta(x)$ and $P \rightarrow_{\mathrm{A}} Q$. We want to show that $Q \in \eta(x)$. As $P \rightarrow_{\mathrm{A}} Q$, there is some $a \in A \backslash A_{\downarrow 2}$ and $\{m, \tilde{m}\}=\{1,2\}$ such that $u_{m}(a) \in P, u_{\tilde{m}}(a) \in Q$ and $a(t)=m$. We must have $u_{m}(x(a))=$ $x\left(u_{m}(a)\right)=u_{m}(a(t))=u_{m}(m)$, since $\left.x\right|_{P}=\left.\pi_{t}\right|_{P}$. Therefore $x(a)=m=a(t)$ and $x\left(u_{\tilde{m}}(a)\right)=u_{\tilde{m}}(x(a))=u_{\tilde{m}}(a(t))$. Since $\mathscr{P}\left(u_{\tilde{m}}(a)\right)$ has two blocks, we have $\pi_{s}\left(u_{\tilde{m}}(a)\right) \neq \pi_{t}\left(u_{\tilde{m}}(a)\right)$ and therefore $x \uparrow_{Q}=\pi_{t} \uparrow_{Q}$. Thus $\eta(x) \in \mathscr{I}_{t}(\mathbf{A})$ and $\eta$ is well defined.

To show that $\eta$ is a bijection, let $Z \in \mathscr{I}_{t}(\mathbf{A})$. As $\left\{P \backslash\{\hat{0}, \widehat{1}, \widehat{2}\} \mid P \in \mathscr{P}_{\mathrm{A}}\right\}$ forms a partition of $A_{\downarrow 2} \backslash\{\widehat{0}, \widehat{1}, \widehat{2}\}$, we can define the homomorphism $x: \mathbf{A}_{\downarrow 2} \rightarrow \underline{\mathbf{M}}$ by

$$
x:=\bigcup\left\{\left.\pi_{t}\right|_{P} \mid P \in Z\right\} \cup \bigcup\left\{\left.\pi_{s}\right|_{P} \mid P \in \mathscr{P}_{\mathbf{A}} \backslash Z\right\} .
$$

We want to prove that $x$ has a unique extension $\bar{x}: \mathbf{A} \rightarrow \underline{\mathbf{M}}$. Let $a \in A \backslash A_{\downarrow 2}$ and let $Q_{m}$ be the subuniverse of $\mathbf{A}_{\downarrow 2}$ determined by $\mathscr{P}\left(u_{m}(a)\right)$, for each $m \in\{1,2\}$. Then $\operatorname{sg}_{\mathbf{A}}(a) \subseteq Q_{1} \cup Q_{2} \cup\{a, 021(a)\}$, since every unary term function of $\underline{M}$ belongs to $F^{\sharp}$. We want to find some $r \in S$ such that $x \uparrow_{Q_{1} \cup Q_{2}}=\left.\pi_{r}\right|_{Q_{1} \cup Q_{2}}$. We shall then define $\bar{x}(a):=a(r)$ and, if $021(a) \in A$, we shall define $\bar{x}(021(a)):=021(a)(r)$. It will follow that $x$ extends to $A$. 
For each $m \in\{1,2\}$, we have $u_{m}(a(s))=\pi_{s}\left(u_{m}(a)\right) \neq \pi_{t}\left(u_{m}(a)\right)=u_{m}(a(t))$. This implies that $\{a(s), a(t)\}=\{1,2\}$. Now define $m:=a(t)$ and $\tilde{m}:=a(s)$. Then $Q_{m} \rightarrow_{\mathrm{A}} Q_{\tilde{m}}$. We can assume that $Q_{\tilde{m}} \in Z$ and $Q_{m} \notin Z$. Choose some $r \in a^{-1}(0)$. We have

$$
u_{m}(a(s))=u_{m}(\tilde{m})=u_{m}(a(r)) \quad \text { and } \quad u_{\tilde{m}}(a(t))=u_{\tilde{m}}(m)=u_{\tilde{m}}(a(r)) .
$$

The elements of $Q_{m} \backslash\{\widehat{0}, \hat{1}, \widehat{2}\}$ all determine the same partition of $S$. So $x \uparrow_{Q_{m}}=$ $\left.\pi_{s}\right|_{Q_{m}}=\pi_{r} \uparrow_{Q_{m}}$ and, similarly, $\left.x\right|_{Q_{\tilde{m}}}=\pi_{t} \uparrow_{Q_{\tilde{m}}}=\pi_{r} \uparrow_{Q_{\tilde{m}}}$. Thus $x$ extends to a homomorphism $\bar{x} \in \mathscr{A}(\mathbf{A}, \underline{\mathbf{M}})$. Since $\left.\pi_{s}\right|_{P} \neq\left.\pi_{i}\right|_{P}$, for all $P \in \mathscr{P}_{\mathbf{A}}$, we must have $\eta(\bar{x})=Z$.

Let $y: \mathbf{A} \rightarrow \underline{\mathbf{M}}$ be an extension of $x$. For all $a \in A \backslash A_{\downarrow 2}$ and $m \in\{1,2\}$, we have

$$
\begin{aligned}
& \bar{x}(a)=m \quad \Longleftrightarrow \quad u_{m}(\bar{x}(a))=u_{m}(m) \quad \Longleftrightarrow \quad \bar{x}\left(u_{m}(a)\right)=u_{m}(m) \\
& \Longleftrightarrow y\left(u_{m}(a)\right)=u_{m}(m) \quad \Longleftrightarrow \quad u_{m}(y(a))=u_{m}(m) \\
& \Longleftrightarrow \quad y(a)=m \text {. }
\end{aligned}
$$

So $\bar{x}$ is the unique extension of $x$ to $\mathbf{A}$. It follows that $\eta$ is a bijection.

We say that the algebra $\mathbf{A} \leqslant \underline{\mathbf{M}}^{S}$ is locally balanced if $x \uparrow_{B}$ is the restriction of a projection, for each homomorphism $x: \mathbf{A} \rightarrow \underline{\mathbf{M}}$ and each finite subset $B$ of $A$. For all $\mathbf{B} \leqslant \underline{\mathbf{M}}^{s}$ and $s \in S$, define the homomorphism $\rho_{s}: \mathbf{B} \rightarrow \underline{\mathbf{M}}$ by $\rho_{s}:=\left.\pi_{s}\right|_{B}$.

LEMMA 5.6. Let $\underline{\mathrm{M}}$ be a unary algebra of type $(2)_{\mathrm{Q}}$ or type $(2)_{\mathrm{C}}$, and define $\mathscr{A}:=\mathbb{\square} \mathbb{P}(\underline{\mathbf{M}})$. Let $\mathbf{B} \leqslant \mathbf{A} \leqslant \underline{\mathbf{M}}^{S}$ such that $A_{\downarrow 2}=B_{\downarrow 2}$ and $\mathbf{A}$ is locally balanced. Define the set $X:=\left\{\left.x\right|_{B} \mid x \in \mathscr{A}(\mathbf{A}, \underline{\mathbf{M}})\right\}$ and let $s, t \in S$. Assume that the relation

$$
\leqslant:=\left\{(x, y) \in X^{2} \mid\left(\rho_{s}, x, y, \rho_{t}\right) \in \preccurlyeq_{4}\right\}
$$

on $X$ is reflexive.

(i) For all $x \in X$ and $P \in \mathscr{P}_{\mathrm{A}}$, we have $x \uparrow_{P}=\pi_{s} \uparrow_{P}$ or $x \uparrow_{P}=\pi_{t} \uparrow_{P}$.

(ii) There is a well-defined order-isomorphism $\eta:\langle X ; \leqslant\rangle \rightarrow\left\langle\mathscr{I}_{t}(\mathbf{A}) ; \subseteq\right\rangle$ given by $\eta(x):=\left\{P \in \mathscr{P}_{\mathrm{A}}|x|_{P}=\pi_{t} \uparrow_{P}\right\}$.

(iii) For all $n \in \mathbb{N}$ and all $x_{1}, \ldots, x_{n} \in X$ such that $x_{1} \leqslant \cdots \leqslant x_{n}$, we have $\left(\rho_{s}, x_{1}, \ldots, x_{n}, \rho_{t}\right) \in \preccurlyeq_{n+2}$.

PROOF. There are unary term functions $u_{1}$ and $u_{2}$ of $\underline{\mathbf{M}}$ with $\operatorname{ker}\left(u_{1}\right)=\{02 \mid 1\}$ and $\operatorname{ker}\left(u_{2}\right)=\{01 \mid 2\}$. We must have $\mathscr{P}_{\mathrm{A}}=\mathscr{P}_{\mathrm{B}}$, since $A_{\downarrow 2}=B_{\downarrow 2}$. To prove (i), let $x \in X$ and $P \in \mathscr{P}_{\mathrm{A}}$. There is some $a \in P$ such that $|\mathscr{P}(a)|=2$. The relation $\leqslant$ is reflexive, so $\left(\rho_{s}, x, x, \rho_{t}\right)(a) \in \preccurlyeq_{4}$. Since $\mathbf{A}$ is locally balanced, the map $x \uparrow_{P}$ is the restriction of a projection. Therefore $|\{x(a), a(s), a(t)\}| \leqslant 2$. By Lemma 5.3 (i), this implies 
that $x(a)=a(s)$ or $x(a)=a(t)$. Since all the elements of $P \backslash\{\widehat{0}, \widehat{1}, \widehat{2}\}$ determine the same partition of $S$, we must have $x \uparrow_{P}=\pi_{s} \uparrow_{P}$ or $x \uparrow_{P}=\pi_{t} \uparrow_{P}$. Thus (i) holds and the map $\eta$, given in (ii), is a well-defined bijection, by Lemma 5.5. (Note that $\eta$ is still a bijection even if $\mathscr{P}_{\mathbf{A}}=\varnothing$ and we cannot use Lemma 5.5, as the algebra $\mathbf{A}$ is locally balanced.)

We now want to show that $\eta$ is an isomorphism. Let $x, y \in X$ and assume that $x \leqslant y$. To see that $\eta(x) \subseteq \eta(y)$, let $P \in \eta(x)$. There is some $a \in P$ such that $|\mathscr{P}(a)|=2$. We have $\left(\rho_{s}, \rho_{t}, y, \rho_{t}\right)(a)=\left(\rho_{s}, x, y, \rho_{t}\right)(a) \in \preccurlyeq_{4}$ and therefore $y(a)=a(t)$, by Lemma 5.3 (i). Thus $P \in \eta(y)$, which implies that $\eta(x) \subseteq \eta(y)$.

Now assume $\eta(x) \subseteq \eta(y)$ and let $b \in B$. We will show that $b(s)=x(b)$ or $x(b)=y(b)$ or $y(b)=b(t)$. It will then follow that $\left(\rho_{s}, x, y, \rho_{t}\right)(b) \in \preccurlyeq_{4}$, since $\left(\rho_{s}, y, y, \rho_{t}\right),\left(\rho_{s}, x, x, \rho_{t}\right) \in \preccurlyeq_{4}$ and therefore $\left(\rho_{s}, \rho_{s}, y, \rho_{t}\right),\left(\rho_{s}, x, \rho_{t}, \rho_{t}\right) \in \preccurlyeq_{4}$, by Lemma 5.3 (ii). Assume that $b(s) \neq x(b)$ and $y(b) \neq b(t)$. There is some $m \in\{1,2\}$ such that $u_{m}(x(b)) \neq u_{m}(b(s))$. Define $Q_{m}$ to be the subuniverse of $\mathbf{A}_{\downarrow 2}$ determined by $\mathscr{P}\left(u_{m}(b)\right)$. Then $x \uparrow_{Q_{m}}=\pi_{t} \uparrow_{Q_{m}}$, by (i). As $\eta(x) \subseteq \eta(y)$, this implies that $y \uparrow_{Q_{m}}=\pi_{t} \uparrow_{Q_{m}}$ and therefore $u_{m}(y(b))=u_{m}(b(t))=u_{m}(x(b))$. Define $\tilde{m}:=021(m)$ and let $Q_{\tilde{m}}$ denote the subuniverse of $\mathbf{A}_{\downarrow 2}$ determined by $\mathscr{P}\left(u_{\tilde{m}}(b)\right)$. We must have $u_{\tilde{m}}(y(b)) \neq u_{\tilde{m}}(b(t))$, since $y(b) \neq b(t)$. Now $\eta(x) \subseteq \eta(y)$ implies that $\left.x\right|_{Q_{\tilde{m}}}=\pi_{s} \Upsilon_{Q_{\tilde{m}}}=y\left\lceil_{Q_{\tilde{m}}}\right.$, and therefore $u_{\tilde{m}}(x(b))=u_{\tilde{m}}(b(s))=u_{\tilde{m}}(y(b))$. So $x(b)=y(b)$, as $u_{1}$ and $u_{2}$ separate $M$. Thus $x \leqslant y$. We have proven that $\eta$ is an isomorphism, and so (ii) holds.

To prove (iii), let $n \in \mathbb{N}$ and $x_{1}, \ldots, x_{n} \in X$ with $x_{1} \leqslant \ldots \leqslant x_{n}$. Let $b \in B$. We will show that there are $j, k \in\{1, \ldots, n\}$, with $j \leqslant k$, such that

$$
x_{i}(b)= \begin{cases}\rho_{s}(b) & \text { if } i<j \\ x_{j}(b) & \text { if } j \leqslant i \leqslant k \\ \rho_{t}(b) & \text { if } k<i\end{cases}
$$

for all $i \in\{1, \ldots, n\}$. As $\leqslant$ is reflexive, it will then follow, by Lemma 5.3 (ii), that $\left(\rho_{s}, x_{1}, \ldots, x_{n}, \rho_{t}\right)(b) \in \preccurlyeq_{n+2}$. Let $i \in\{1, \ldots, n\}$. If $i \neq n$ and $x_{i}(b)=\rho_{t}(b)$, then $\left(\rho_{s}, \rho_{t}, x_{i+1}, \rho_{t}\right)(b)=\left(\rho_{s}, x_{i}, x_{i+1}, \rho_{t}\right)(b) \in \preccurlyeq_{4}$ and therefore $x_{i+1}(b)=\rho_{t}(b)$, by Lemma 5.3 (i). Similarly, if $i \neq 1$ and $x_{i}(b)=\rho_{s}(b)$, then $x_{i-1}(b)=\rho_{s}(b)$. Since the algebra $\mathbf{A}$ is locally balanced, we know that $|\{x(b) \mid x \in X\}| \leqslant 3$. Thus (iii) holds.

For each ordered set $\langle X ; \leqslant\rangle$, define $\mathscr{L}_{\leqslant}(X)$ to be the set of all $x \in X$ that have a unique lower cover $x^{\downarrow}$ in $\langle X ; \leqslant\rangle$.

LEMMA 5.7. Let $\underline{\mathrm{M}}$ be a unary algebra of type $(2)_{\mathrm{Q}}$ or type $(2)_{\mathrm{C}}$, and define $\mathscr{A}:=\operatorname{ISP}(\underline{\mathbf{M}})$. Let $\mathbf{B} \leqslant \mathbf{A} \leqslant \underline{\mathbf{M}}^{s}$ such that $A_{\downarrow 2}=B_{\downarrow 2}$ and $\mathbf{A}$ is locally balanced. 
Define the set $X:=\left\{\left.x\right|_{B} \mid x \in \mathscr{A}(\mathbf{A}, \underline{\mathbf{M}})\right\}$ and let $s, t \in S$. Assume that

$$
\leqslant:=\left\{(x, y) \in X^{2} \mid\left(\rho_{s}, x, y, \rho_{t}\right) \in \preccurlyeq_{4}\right\}
$$

is an order on $X$ and define

$$
\geqslant^{*}:=\left\{(x, y) \in \mathscr{L}_{\leqslant}(X)^{2} \mid x \geqslant y \text { and }\left(\rho_{s}, y^{\downarrow}, y, x^{\downarrow}, x, \rho_{t}\right) \notin \bowtie\right\} .
$$

Then the structures $\left\langle\mathscr{L}_{\leqslant}(X) ; \geqslant *, \geqslant\right\rangle$ and $\left(\mathscr{P}_{\mathbf{A}} ; \rightarrow_{t}, \rightarrow_{\mathbf{B}} \rightarrow_{\mathbf{A}}\right\rangle$ are isomorphic.

PROOF. There are unary term functions $u_{1}$ and $u_{2}$ of $\underline{\mathbf{M}}$ with $\operatorname{ker}\left(u_{1}\right)=\{02 \mid 1\}$ and $\operatorname{ker}\left(u_{2}\right)=\{01 \mid 2\}$. We must have $\mathscr{P}_{\mathrm{A}}=\mathscr{P}_{\mathrm{B}}$, since $A_{\downarrow 2}=B_{\downarrow 2}$. Lemma 5.6 tells us that $x \uparrow_{P}=\pi_{s} \uparrow_{P}$ or $x \uparrow_{P}=\pi_{t} \uparrow_{P}$, for all $x \in X$ and $P \in \mathscr{P}_{\mathrm{A}}$, and also that the map $\eta:\langle X ; \leqslant\rangle \rightarrow\left\langle\mathscr{I}_{t}(\mathbf{A}) ; \subseteq\right\rangle$, given by $\eta(x):=\left\{P \in \mathscr{P}_{\mathbf{A}}|x|_{P}=\left.\pi_{t}\right|_{P}\right\}$, is an order-isomorphism.

For each $P \in \mathscr{P}_{\mathrm{A}}$, define the $\rightarrow_{t} \mathrm{~A}^{\text {-increasing subset }}$

$$
Z_{P}:=\left\{Q \in \mathscr{P}_{\mathrm{A}} \mid P \rightarrow_{\mathrm{A}} Q\right\}
$$

of $\mathscr{P}_{\mathrm{A}}$. Then $\mathscr{L}_{\subseteq}\left(\mathscr{I}_{1}(\mathbf{A})\right)=\left\{Z_{P} \mid P \in \mathscr{P}_{\mathrm{A}}\right\}$ and so we can define $\zeta: \mathscr{P}_{\mathrm{A}} \rightarrow \mathscr{L}_{\leqslant}(X)$ by $\zeta(P):=\eta^{-1}\left(Z_{P}\right)$. Since $\eta$ is an isomorphism, the map $\zeta$ is onto. To see that $\zeta$ is one-to-one, suppose that $P, Q \in \mathscr{P}_{\mathrm{A}}$ such that $P \neq Q$ and $\zeta(P)=\zeta(Q)$. Then $Z_{P}=Z_{Q}$. For all $x \in X$, we have $\left.x\right|_{P \cup Q}=\left.\pi_{s}\right|_{P \cup Q}$ or $\left.x\right|_{P \cup Q}=\pi_{t} \uparrow_{P \cup Q}$, as $\eta(x) \in \mathscr{I}_{1}(\mathbf{A})$. This is a contradiction, since $P$ and $Q$ determine different partitions of $S$. Thus $\zeta$ is a bijection.

We will show that $\zeta$ is an isomorphism between the structures $\left\langle\mathscr{P}_{\mathbf{A}} ; \rightarrow_{\boldsymbol{B}}, \rightarrow_{\mathbf{A}}\right\rangle$ and $\left\langle\mathscr{L}_{\leqslant}(X) ; \geqslant *, \geqslant\right\rangle$. Let $P, Q \in \mathscr{P}_{\mathrm{A}}$ such that $P \neq Q$. Define $x_{P}:=\zeta(P)$ and $x_{Q}:=\zeta(Q)$. Then

$$
P \underset{\mathrm{r}}{\rightarrow} Q \Longleftrightarrow Z_{P} \supseteq Z_{Q} \Longleftrightarrow \eta^{-1}\left(Z_{P}\right) \geqslant \eta^{-1}\left(Z_{Q}\right) \Longleftrightarrow x_{P} \geqslant x_{Q} .
$$

So $\rightarrow_{t}$ is an order on $\mathscr{P}_{\mathbf{A}}$.

By Lemma 5.3 (i), the relation $\geqslant^{*}$ on $\mathscr{L}_{\leqslant}(X)$ is reflexive. So it remains to prove that $P \rightarrow_{\mathrm{B}} Q$ if and only if $x_{P} \geqslant^{*} x_{Q}$. First assume that $P \rightarrow_{\mathrm{B}} Q$. There is some $b \in B \backslash B_{\downarrow 2}$ and $\{m, \tilde{m}\}=\{1,2\}$ with $u_{m}(b) \in P, u_{\tilde{m}}(b) \in Q$ and $b(t)=m$. We have $x \uparrow_{Q}=\pi_{s} \uparrow_{Q}$ or $x \uparrow_{Q}=\left.\pi_{t}\right|_{Q}$, for all $x \in X$. So $u_{\tilde{m}}(b(s)) \neq u_{\tilde{m}}(b(t))=u_{\tilde{m}}(m)$ and therefore $b(s)=\tilde{m}$. Since $\rightarrow_{t}$ is an order on $\mathscr{P}_{\mathrm{A}}$, we have $\eta\left(x_{P}^{\downarrow}\right)=Z_{P}^{\downarrow}=Z_{P} \backslash\{P\}$ and $\eta\left(x_{Q}^{t}\right)=Z_{Q}^{\downarrow}=Z_{Q} \backslash\{Q\}$. As $P \longrightarrow \rightarrow_{\mathrm{A}} Q$, it follows that

$$
\begin{aligned}
& u_{m}\left(x_{Q}^{\downarrow}(b)\right)=x_{Q}^{\downarrow}\left(u_{m}(b)\right)=u_{m}(b(s))=u_{m}(\tilde{m}), \\
& u_{\tilde{m}}\left(x_{Q}^{\downarrow}(b)\right)=x_{Q}^{\downarrow}\left(u_{\tilde{m}}(b)\right)=u_{\tilde{m}}(b(s))=u_{\tilde{m}}(\tilde{m}),
\end{aligned}
$$


and, similarly,

$$
\begin{array}{ll}
u_{m}\left(x_{Q}(b)\right)=u_{m}(b(s))=u_{m}(0), & u_{\tilde{m}}\left(x_{Q}(b)\right)=u_{\tilde{m}}(b(t))=u_{\tilde{m}}(0) \\
u_{m}\left(x_{P}^{\dagger}(b)\right)=u_{m}(b(s))=u_{m}(0), & u_{\tilde{m}}\left(x_{P}^{\downarrow}(b)\right)=u_{\tilde{m}}(b(t))=u_{\tilde{m}}(0), \\
u_{m}\left(x_{P}(b)\right)=u_{m}(b(t))=u_{m}(m), & u_{\tilde{m}}\left(x_{P}(b)\right)=u_{\tilde{m}}(b(t))=u_{\tilde{m}}(m) .
\end{array}
$$

This implies that $\left(\rho_{s}, x_{Q}^{\mathfrak{t}}, x_{Q}, x_{P}^{\downarrow}, x_{P}, \rho_{t}\right)(b)=(\tilde{m}, \tilde{m}, 0,0, m, m)$, since $u_{1}$ and $u_{2}$ separate the elements of $M$. So $\left(\rho_{s}, x_{Q}^{\downarrow}, x_{Q}, x_{P}^{\downarrow}, x_{P}, \rho_{t}\right) \notin \bowtie$, by Lemma 5.3 (iii). As $P \rightarrow \rightarrow_{\mathrm{A}} Q$ and therefore $x_{P} \geqslant x_{Q}$, we have shown that $x_{P} \geqslant * x_{Q}$.

Now assume that $x_{P} \geqslant * x_{Q}$. Then $x_{Q}^{\downarrow} \leqslant x_{Q} \leqslant x_{P}^{\downarrow} \leqslant x_{P}$, which implies that $\left(\rho_{s}, x_{Q}^{\downarrow}, x_{Q}, x_{P}^{\downarrow}, x_{P}, \rho_{t}\right) \in \preccurlyeq_{6}$, by Lemma 5.6 (iii). Since $\left(\rho_{s}, x_{Q}^{\downarrow}, x_{Q}, x_{P}^{\downarrow}, x_{P}, \rho_{t}\right) \notin \bowtie$, there is some $b \in B$ and $\{m, \widetilde{m}\}=\{1,2\}$ such that $\left(\rho_{s}, x_{Q}^{\downarrow}, x_{Q}, x_{P}^{\downarrow}, x_{P}, \rho_{t}\right)(b)=$ ( $\tilde{m}, \tilde{m}, 0,0, m, m)$, by Lemma 5.3 (iii). As $\mathbf{A}$ is locally balanced, this implies that $b \notin B_{\downarrow 2}$. We have

$$
\begin{array}{ll}
x_{P}^{\downarrow}\left(u_{m}(b)\right)=u_{m}(0)=u_{m}(b(s)), & x_{Q}^{\downarrow}\left(u_{\tilde{m}}(b)\right)=u_{\tilde{m}}(\tilde{m})=u_{\tilde{m}}(b(s)), \\
x_{P}\left(u_{m}(b)\right)=u_{m}(m)=u_{m}(b(t)), & x_{Q}\left(u_{\tilde{m}}(b)\right)=u_{\tilde{m}}(0)=u_{\tilde{m}}(b(t)) .
\end{array}
$$

It follows that the subuniverse of $\mathbf{A}_{\downarrow 2}$ determined by $\mathscr{P}\left(u_{m}(b)\right)$ belongs to $Z_{P}$ but not to $Z_{P} \backslash\{P\}$. Therefore $u_{m}(b) \in P$ and, similarly, $u_{\tilde{m}}(b) \in Q$. Thus $P \rightarrow_{\mathbf{B}} Q$, whence $\zeta$ is an isomorphism.

The next lemma will complete the preparation for our proof that algebras with type $(2)_{Q}$ or type $(2)_{C}$ are not fully dualisable. The algebras $\overparen{\Gamma}$ and $\widehat{\Gamma}_{\triangleleft}$ come from Lemma 4.1 and Definition 4.3.

LEMMA 5.8. Let $\underline{\mathbf{M}}$ be a unary algebra with type $(2)_{\mathrm{Q}}$ and define $\mathscr{A}:=\mathbb{I S P}(\underline{\mathrm{M}})$. Then $\widehat{\Gamma}_{\triangleleft} \leqslant \widehat{\Gamma} \leqslant \underline{\mathbf{M}}^{\Gamma^{+}}$such that $(\widehat{\Gamma})_{\downarrow 2}=\left(\widehat{\Gamma}_{\triangleleft}\right)_{\downarrow 2}$ and $\widehat{\Gamma}$ is locally balanced. Define the set $X:=\left\{x\left\lceil\widehat{T}_{\triangleleft} \mid x \in \mathscr{A}(\widehat{\Gamma}, \underline{\mathbf{M}})\right\}\right.$. Then the relation

$$
\leqslant:=\left\{(x, y) \in X^{2} \mid\left(\rho_{\top}, x, y, \rho_{\perp}\right) \in \preccurlyeq_{4}\right\}
$$

on $X$ is reflexive. The structures $\left\langle\mathscr{P}_{\widehat{\Gamma}} ; \underset{\perp}{\longrightarrow} \widehat{\Gamma}_{\triangleleft}, \longrightarrow \longrightarrow_{\perp}\right\rangle$ and $\langle\Gamma ; \triangleleft, \leqslant\rangle$ are isomorphic.

Proof. It follows by Lemma 4.4 (ii) that $(\overparen{\Gamma})_{\downarrow 2}=\left(\widehat{\Gamma}_{\triangleleft}\right)_{\downarrow 2}$. To see that $\overparen{\Gamma}$ is locally balanced, let $x \in \mathscr{A}(\widehat{\Gamma}, \underline{\mathbf{M}})$ and let $B \subseteq \widehat{\Gamma}$ be finite. There is a finite subset $\Gamma_{0}$ of $\Gamma$ such that $B \subseteq \operatorname{sg}_{\overparen{\Gamma}}\left(\left\{\widehat{a b} \mid a, b \in \Gamma_{0}\right.\right.$ and $\left.\left.a \leqslant b\right\}\right)$. The map $x \circ \iota_{\Gamma}: \Gamma \rightarrow \underline{\mathbf{2}}$ is order preserving, by Lemma 4.6 (ii). First assume that $x(\overparen{b b})=1$, for all $b \in \Gamma_{0}$. By Lemma 4.6 (i), we have $x(\overparen{a b})=1$, for all $a, b \in \Gamma_{0}$ with $a \leqslant b$, and therefore 
$x \uparrow_{B}=\pi_{\mathrm{T}} \uparrow_{B}$. Now we can assume that there is a minimum element $c$ of $\Gamma_{0}$ in $\Gamma$ such that $x(\widehat{c c})=2$. For all $a, b \in \Gamma_{0}$ with $a \leqslant b$, we have

$$
\begin{aligned}
x(\widehat{a b}) & = \begin{cases}2 & \text { if } c \leqslant a \\
0 & \text { if } a<c \leqslant b ; \\
1 & \text { if } b<c,\end{cases} \\
& =\widehat{a b}(c),
\end{aligned}
$$

by Lemma 4.6 (i). Thus $x \uparrow_{B}=\pi_{C} \uparrow_{B}$ and $\widehat{\Gamma}$ is locally balanced.

For all $a, b \in \Gamma$ with $a \triangleleft b$ and all $x \in X$, we have $\rho_{\top}(\widehat{a b})=1 \preccurlyeq x(\widehat{a b}) \preccurlyeq 2=$ $\rho_{\perp}(\widehat{a b})$ and therefore $\left(\rho_{\top}, x, x, \rho_{\perp}\right)(\widehat{a b}) \in \preccurlyeq_{4}$. So $\leqslant$ is reflexive. It is easy to check that $\left\langle\mathscr{P}_{\mathrm{T}} ; \longrightarrow \widehat{\mathrm{T}}_{\triangleleft}, \longrightarrow{ }_{\mathrm{T}}\right\rangle$ and $\langle\Gamma ; \triangleleft, \leqslant\rangle$ are isomorphic; see Example 5.4.

An algebra $\mathbf{A} \leqslant \underline{\mathbf{M}}^{S}$ is called balanced if $\mathscr{A}(\mathbf{A}, \underline{\mathbf{M}})=\left\{\left.\pi_{s}\right|_{A} \mid s \in S\right\}$, and $\left.\pi_{s}\right|_{A} \neq \pi_{i} \uparrow_{A}$, for all $s, t \in S$ with $s \neq t$. It is easy to check that every algebra in $\mathbb{D S P}(\underline{\mathrm{M}})$ is isomorphic to a balanced algebra.

THEOREM 5.9. Let $\underline{\mathrm{M}}$ be a unary algebra of type $(2)_{\mathrm{Q}}$ or type (2) $\mathrm{C}_{\mathrm{C}}$. Then $\underline{\mathrm{M}}$ is not fully dualisable.

PROOF. First assume that $\underline{\mathbf{M}}$ has type $(2)_{\mathrm{Q}}$. Suppose there is an alter ego $\mathbf{\sim}$ of $\underline{\mathbf{M}}$ that yields a full duality on $\mathscr{A}:=\mathbb{S \mathbb { P }}(\underline{\mathrm{M}})$. By Lemma 5.1 and Lemma 5.2 , we can assume that $\preccurlyeq_{4}$ and $\bowtie$ are in the type of $\mathbf{M}$. Using Lemma 4.1 and Definition 4.3, there are algebras $\widehat{\boldsymbol{\Gamma}}_{\triangleleft}$ and $\widetilde{\boldsymbol{\Gamma}}$ in $\mathscr{A}$. The set $X:=\left\{x \widehat{\Gamma}_{\triangleleft} \mid x \in \mathscr{A}(\widehat{\boldsymbol{\Gamma}}, \underline{\mathbf{M}})\right\}$ forms a closed substructure of $\underset{\sim}{\mathbf{M}}{ }^{P}$, by Lemma 4.2 (i) and Lemma 4.6 (ii). Since $\mathbf{\sim}$ yields a full duality on $\mathscr{A}$, there is an isomorphism $\varphi: \mathbf{X} \rightarrow \mathrm{D}(\mathbf{A})$, for some balanced algebra $\mathrm{A} \leqslant \underline{\mathbf{M}}^{S}$, with $S$ a non-empty set.

As $\mathbf{A}$ is balanced, there exist $s, t \in S$ with $\varphi\left(\rho_{\top}\right)=\rho_{s}$ and $\varphi\left(\rho_{\perp}\right)=\rho_{t}$. By Lemma 5.6 (ii) and Lemma 5.8, the relation $\leqslant:=\left\{(x, y) \in X^{2} \mid\left(\rho_{\mathrm{T}}, x, y, \rho_{\perp}\right) \in \preccurlyeq_{4}\right\}$ is an order on $X$. Since $\varphi$ is an isomorphism and $\preccurlyeq_{4}$ is in the type of $\underset{\sim}{\mathbf{M}}$, we have

$$
\varphi(\leqslant)=\left\{(x, y) \in \mathscr{A}(\mathbf{A}, \underline{\mathbf{M}})^{2} \mid\left(\rho_{s}, x, y, \rho_{t}\right) \in \preccurlyeq_{4}\right\} .
$$

Define

$$
\geqslant^{*}:=\left\{(x, y) \in \mathscr{L}_{\leqslant}(X)^{2} \mid x \geqslant y \text { and }\left(\rho_{\mathrm{T}}, y^{\downarrow}, y, x^{\downarrow}, x, \rho_{\perp}\right) \notin \bowtie\right\} .
$$

Then $\varphi\left(\geqslant^{*}\right)=\left\{(x, y) \in \mathscr{L}_{\varphi(\leqslant)}(\mathscr{A}(\mathbf{A}, \underline{\mathbf{M}}))^{2} \mid x \varphi(\geqslant) y\right.$ and $\left.\left(\rho_{s}, y^{\downarrow}, y, x^{\downarrow}, x, \rho_{t}\right) \notin \bowtie\right\}$. Using Lemma 5.7 twice and Lemma 5.8, it follows that

$$
\begin{aligned}
& \left\langle\mathscr{P}_{\mathbf{A}} ; \rightarrow_{\mathbf{i}}, \rightarrow_{\boldsymbol{t}}\right\rangle \cong\left\langle\mathscr{L}_{\varphi(\leqslant)}(\mathscr{A}(\mathbf{A}, \underline{\mathbf{M}})) ; \varphi\left(\geqslant^{*}\right), \varphi(\geqslant)\right\rangle \\
& \cong\left\langle\mathscr{L}_{\leqslant}(X) ; \geqslant *, \geqslant\right\rangle \\
& \cong\left\langle\mathscr{P}_{\mathrm{\Gamma}} ; \longrightarrow_{\mathrm{\Gamma}}, \longrightarrow \underset{\mathrm{I}}{\longrightarrow}\right\rangle \text {. }
\end{aligned}
$$


So $\left\langle\mathscr{P}_{\mathbf{A}} ; \rightarrow_{\mathbf{A}}, \rightarrow_{\mathbf{A}}\right\rangle$ is isomorphic to $\langle\Gamma ; \triangleleft, \leqslant\rangle$, by Lemma 5.8. But this implies that $\leqslant$ is the transitive closure of $\triangleleft$, which is a contradiction. Thus $\underline{\mathbf{M}}$ is not fully dualisable.

Now assume that $\underline{\mathbf{M}}$ has type $(2)_{\mathrm{C}}$ but does not have type $(2)_{\mathrm{Q}}$. Recall that $\underline{\mathbf{M}}^{\sharp}=\left\langle\{0,1,2\} ; F^{\sharp}\right\rangle$. We can show that $\underline{\mathbf{M}}$ is not fully dualisable, using Lemma 4.8, by following the proof given above with the algebras $\widehat{\boldsymbol{\Gamma}}_{\triangleleft}^{b}$ and $\widehat{\boldsymbol{\Gamma}}^{\mathrm{b}}$ in $\mathscr{A}$, which are the reducts of the algebras $\widehat{\Gamma}_{\triangleleft}$ and $\overparen{\Gamma}$ in $\mathbb{S S \mathbb { P }}\left(\underline{\mathbf{M}}^{\sharp}\right)$.

\section{References}

[1] D. M. Clark and B. A. Davey, Natural dualities for the working algebraist (Cambridge University Press, Cambridge, 1998).

[2] D. M. Clark, P. M. Idziak, L. R. Sabourin, C. Szabó and R. Willard, 'Natural dualities for quasivarieties generated by a finite commutative ring', Algebra Universalis 46 (2001), 285-320.

[3] D. M. Clark, B. A. Davey and J. G. Pitkethly, 'Binary homomorphisms and natural dualities', J. Pure Appl. Algebra 169 (2002), 1-28.

[4] - 'The complexity of dualisability: three-element unary algebras', Internat. J. Algebra Comput., to appear.

[5] B. A. Davey, 'Dualisability in general and endodualisability in particular', in: Logic and algebra (Pontignano, 1994) (eds. A. Ursini and P. Aglianò), Lecture Notes in Pure and Appl. Math. 180 (Marcel Dekker, New York, 1996) pp. 437-455.

[6] J. Hyndman, 'Mono-unary algebras are strongly dualizable', J. Austral. Math. Soc. 72 (2002), 161-172.

[7] J. Hyndman and R. Willard, 'An algebra that is dualizable but not fully dualizable', J. Pure Appl. Algebra 151 (2000), 31-42.

[8] W. A. Lampe, G. F. McNulty and R. Willard, 'Full duality among graph algebras and flat graph algebras', Algebra Universalis 45 (2001), 311-334.

[9] H. A. Priestley, 'Ordered sets and duality for distributive lattices', in: Orders, descriptions and roles (eds. M. Pouzet and D. Richard), Ann. Discrete Math. 23 (North-Holland, Amsterdam, 1984) pp. 39-60.

[10] R. Willard, 'New tools for proving dualizability', in: Dualities, interpretability and ordered structures (eds. J. Vaz de Carvalho and I. Ferreirim), (C.A.U.L., Lisbon, 1999) pp. 69-74.

La Trobe University

Victoria 3086

Australia

e-mail: janegp@alphalink.com.au 
